\title{
SMITH THEORY AND IRREDUCIBLE HOLOMORPHIC SYMPLECTIC MANIFOLDS
}

\author{
SAMUEL BOISSIÈRE, MARC NIEPER-WISSKIRCHEN, AND ALESSANDRA SARTI
}

\begin{abstract}
We study the cohomological properties of the fixed locus $X^{G}$ of an automorphism group $G$ of prime order $p$ acting on a variety $X$ whose integral cohomology is torsion-free. We obtain a precise relation between the mod $p$ cohomology of $X^{G}$ and natural invariants for the action of $G$ on the integral cohomology of $X$. We apply these results to irreducible holomorphic symplectic manifolds of deformation type of the Hilbert scheme of two points on a K3 surface: the main result of this paper is a formula relating the dimension of the mod $p$ cohomology of $X^{G}$ with the rank and the discriminant of the invariant lattice in the second cohomology space with integer coefficients of $X$.
\end{abstract}

\section{INTRODUCTION}

Smith theory is the study of the cohomological properties of a group $G$ of prime order $p$ acting on a topological space $X$. The first important results were obtained by Smith in the late 1930's by the introduction of the so-called Smith cohomology groups and sequences (see Bredon [10]). The use of equivariant cohomology to reformulate Smith theory was begun by Borel 8 in the 1950's and further formalized as the "localisation theorem" of Borel-Atiyah-Segal-Quillen in the 1960's (see Dwyer-Wilkerson [14]).

In this paper, we use these ideas to relate the dimension of the mod $p$ cohomology of the fixed point set $X^{G}$ to natural invariants for the action of $G$ on the integral cohomology $H^{*}(X, \mathbb{Z})$ for $2 \leq p \leq 19$ (see Corollaries [5.11] \& 5.12). This applies nicely to the study of prime order automorphisms on some symplectic holomorphic varieties, particularly those in the deformation class of the Hilbert scheme $S^{[2]}$ of two points on a K3 surface $S$. The first main result of this paper is a degeneracy condition for the spectral sequence of equivariant cohomology

$$
E_{2}^{r, s}:=H^{r}\left(G ; H^{s}\left(X, \mathbb{F}_{p}\right)\right) \Longrightarrow H_{G}^{r+s}\left(X, \mathbb{F}_{p}\right) .
$$

Theorem 1.1. Let $G$ be a group of prime order $p$ acting by automorphisms on an irreducible holomorphic symplectic variety $X$. The spectral sequence of equivariant cohomology with coefficients in $\mathbb{F}_{p}$ degenerate at the $E_{2}$-term in the following cases:

(1) $X$ is deformation equivalent to the Hilbert scheme $S^{[2]}$ of two points on a K3 surface $S$ and $p \notin\{2,5,23\}$.

(2) $X=S^{[2]}, G$ acts by natural automorphisms (induced by automorphisms of the surface $S$ ) and $p \neq 2$.

Date: September 12, 2018.

1991 Mathematics Subject Classification. Primary 14J50; Secondary 14C50, 55T10.

Key words and phrases. Smith theory, holomorphic symplectic manifolds, automorphisms. 
This result is proven in Proposition 6.12 as a consequence of Deligne's criterium (see Section 4.3) applied to specific geometrical objects in the cohomology of $S^{[2]}$ (Lemma 6.9, 6.10 \& 6.11).

For $X$ deformation equivalent to $S^{[2]}$, denote by $\mathrm{T}_{G}(X):=H^{2}(X, \mathbb{Z})^{G}$ the invariant lattice and by $\mathrm{S}_{G}(X):=\mathrm{T}_{G}(X)^{\perp}$ its orthogonal complement for the Beauville-Bogomolov bilinear form. We define (see Definitions 5.5\& 5.9]) two integers $\mathrm{a}_{G}(X), \mathrm{m}_{G}(X) \in \mathbb{N}$ with the property that

$$
\frac{H^{2}(X, \mathbb{Z})}{\mathrm{T}_{G}(X) \oplus \mathrm{S}_{G}(X)} \cong\left(\frac{\mathbb{Z}}{p \mathbb{Z}}\right)^{\mathrm{a}_{G}(X)}, \quad \operatorname{rank} \mathrm{S}_{G}(X)=\mathrm{m}_{G}(X)(p-1) .
$$

The second main result of this paper is the following formula:

Theorem 1.2. Let $X$ be deformation equivalent to $S^{[2]}$ and $G$ be a group of automorphisms of prime order $p$ on $X$ with $3 \leq p \leq 19, p \neq 5$. Then:

$$
\begin{aligned}
\operatorname{dim} H^{*}\left(X^{G}, \mathbb{F}_{p}\right) & =324-2 \mathrm{a}_{G}(X)\left(25-\mathrm{a}_{G}(X)\right)-(p-2) \mathrm{m}_{G}(X)\left(25-2 \mathrm{a}_{G}(X)\right) \\
& +\frac{1}{2} \mathrm{~m}_{G}(X)\left((p-2)^{2} \mathrm{~m}_{G}(X)-p\right)
\end{aligned}
$$

with

$$
\begin{aligned}
& 2 \leq(p-1) \mathrm{m}_{G}(X)<23 \\
& 0 \leq \mathrm{a}_{G}(X) \leq \min \left\{(p-1) \mathrm{m}_{G}(X), 23-(p-1) \mathrm{m}_{G}(X)\right\}
\end{aligned}
$$

This formula is proven in Corollary 6.15. The proof uses first the localisation theorem as presented in Allday-Puppe [1] (see Proposition 4.2), secondly the degeneracy conditions for the spectral sequence of equivariant cohomology with coefficients in $\mathbb{F}_{p}$, then the determination of the $\mathbb{Z}[G]$-module structure of the cohomology space $H^{*}(X, \mathbb{Z})$ (Proposition $[.1$ ), and finally the computation of the quotient $H^{4}(X, \mathbb{Z}) / \operatorname{Sym}^{2} H^{2}(X, \mathbb{Z})$ (Proposition 6.6). The relation with the discriminant of the invariant lattice and its orthogonal is given in Lemma 6.5.

As an application of our results, we show in Section 6.5 that there are no free actions by finite groups on deformations of $S^{[2]}$, and we study an order eleven automorphism on a Fano variety of lines of a cubic fourfold constructed by Mongardi [31].

Aknowledgements. We thank Olivier Debarre, Alexandru Dimca, William G. Dwyer, Viacheslav Kharlamov, Giovanni Mongardi, Kieran O'Grady and Volker Puppe for useful discussions and helpful comments.

\section{TeRminology AND nOtATion}

Let $p$ be a prime number and $G$ a finite cyclic group of order $p$. We fix a generator $g$ of $G$. Put $\tau:=g-1 \in \mathbb{Z}[G]$ and $\sigma:=1+g+\cdots+g^{p-1} \in \mathbb{Z}[G]$.

Let $M$ be a finite-dimensional $\mathbb{F}_{p}$-vector space equipped with a linear action of $G$ (a $\mathbb{F}_{p}[G]$-module for short). The minimal polynomial of $g$, as an endomorphism of $M$, divides the polynomial $X^{p}-1=(X-1)^{p} \in \mathbb{F}_{p}[X]$ hence $g$ admits a Jordan normal form. We can thus decompose $M$ as a direct sum of some $G$-modules $N_{q}$ of dimension $q$ for $1 \leq q \leq p$, where $g$ acts on $N_{q}$ by a matrix (in a suitable basis) of 
the following form:

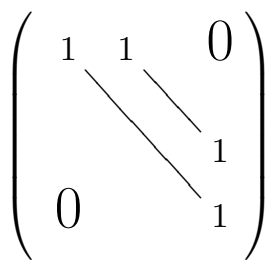

Observe that $N_{p}$ is isomorphic to $\mathbb{F}_{p}[G]$ as a $G$-module. Throughout this paper, the notation $N_{q}$ will always denote the $\mathbb{F}_{p}[G]$-module defined by the Jordan matrix of dimension $q$ above. We define the integer $\ell_{q}(M)$ as the number of blocks of length $q$ in the Jordan decomposition of the $G$-module $M$, in such a way that $M \cong \bigoplus_{q=1}^{p} N_{q}^{\oplus \ell_{q}(M)}$.

Let $H:=\bigoplus_{k>0} H^{k}$ be a finite-dimensional graded $\mathbb{F}_{p}$-vector space, where each graded component $H^{k}$ is equipped with a linear action of $G$. We define similarly, for any $k \geq 0$ and $1 \leq q \leq p$, the integer $\ell_{q}^{k}(H)$ as the number of blocks of length $q$ in the Jordan decomposition of the $G$-module $H^{k}$.

For any topological space $Y$ with the homotopy type of a finite $\mathrm{CW}$-complex and any field $\mathbb{K}$, we set $h^{k}(Y, \mathbb{K}):=\operatorname{dim}_{\mathbb{K}} H^{k}(Y, \mathbb{K})$ and $h^{*}(Y, \mathbb{K}):=\sum_{k \geq 0} h^{k}(Y, \mathbb{K})$.

Let $X$ be a smooth connected orientable compact real even-dimensional manifold, with a smooth orientation-preserving action of $G$. Denote by $X^{G} \subset X$ the fixed locus of $X$ for the action of $G$; then $X^{G}$ is a smooth submanifold of $X$. We define the integers $\ell_{q}^{k}(X)$ for $1 \leq q \leq p$ and $0 \leq k \leq \operatorname{dim}_{\mathbb{R}} X$ as the number of blocks of length $q$ in the Jordan decomposition of the $G$-modules $H^{k}\left(X, \mathbb{F}_{p}\right)$ and we set $\ell_{q}^{*}(X):=\sum_{k \geq 0} \ell_{q}^{k}(X)$.

\section{Some useful Computations in GROUP COHOMOLOGY}

There is a projective resolution $F_{*} \stackrel{\epsilon}{\rightarrow} \mathbb{Z}$ of $\mathbb{Z}$ considered as a $G$-module with a trivial action, given by:

$$
\cdots \longrightarrow \mathbb{Z}[G] \stackrel{\tau}{\rightarrow} \mathbb{Z}[G] \stackrel{\sigma}{\rightarrow} \mathbb{Z}[G] \stackrel{\tau}{\rightarrow} \mathbb{Z}[G] \stackrel{\epsilon}{\rightarrow} \mathbb{Z} \longrightarrow 0
$$

where $\epsilon$ is the summation map: $\epsilon\left(\sum_{j=0}^{p-1} \alpha_{j} g^{j}\right)=\sum_{j=0}^{p-1} \alpha_{j}$ and $\tau, \sigma$ act by multiplication.

Let $\mathbb{F}_{p}:=\mathbb{Z} / p \mathbb{Z}$ considered as a trivial $G$-module. The cohomology groups of $G$ with coefficients in $\mathbb{F}_{p}$ are the cohomology groups of the complex:

$$
0 \rightarrow \operatorname{Hom}_{G}\left(\mathbb{Z}[G], \mathbb{F}_{p}\right) \stackrel{\tau^{*}}{\rightarrow} \operatorname{Hom}_{G}\left(\mathbb{Z}[G], \mathbb{F}_{p}\right) \stackrel{\sigma^{*}}{\rightarrow} \operatorname{Hom}_{G}\left(\mathbb{Z}[G], \mathbb{F}_{p}\right) \stackrel{\tau^{*}}{\rightarrow} \cdots
$$

Observe that $\operatorname{Hom}_{G}\left(\mathbb{Z}[G], \mathbb{F}_{p}\right) \cong \mathbb{F}_{p}$ by identifying a $G$-homomorphism $c$ with its image $c(1) \in \mathbb{F}_{p}$, so $\tau^{*}$ and $\sigma^{*}$ are identically zero and we get $H^{i}\left(G ; \mathbb{F}_{p}\right) \cong \mathbb{F}_{p}$ for all $i \geq 0$.

Let now $M$ be as before a $\mathbb{F}_{p}[G]$-module of finite dimension over $\mathbb{F}_{p}$. The cohomology of $G$ with coefficients in $M$ can be computed in a similar way as the cohomology of the complex:

$$
0 \rightarrow M \stackrel{\bar{\tau}}{\rightarrow} M \stackrel{\bar{\sigma}}{\rightarrow} M \stackrel{\bar{\tau}}{\rightarrow} \cdots
$$

where $\bar{\tau}, \bar{\sigma} \in \mathbb{F}_{p}[G]$ denote the reduction modulo $p$ of $\tau$ and $\sigma$. Observe that

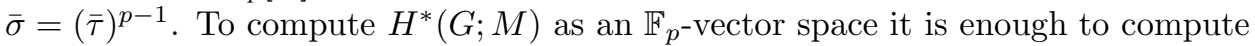
the groups $H^{*}\left(G ; N_{q}\right)$. 


\section{Lemma 3.1.}

(1) If $q<p$ then $H^{i}\left(G ; N_{q}\right)=\mathbb{F}_{p}$ for all $i \geq 0$.

(2) $H^{0}\left(G ; N_{p}\right)=\mathbb{F}_{p}$ and $H^{i}\left(G ; N_{p}\right)=0$ for all $i \geq 1$.

Proof. The case $q=1$ is clear since $N_{1} \cong \mathbb{F}_{p}$ as a trivial $G$-module. Assume now that $q \geq 2$. Let $v_{1}, \ldots, v_{q}$ be a basis of $N_{q}$ such that $g v_{1}=v_{1}$ and $g v_{i}=v_{i-1}+v_{i}$ for all $i \geq 2$. It is easy to compute that, as endomorphisms of $N_{q}$, one has $\operatorname{ker}(\bar{\tau})=\left\langle v_{1}\right\rangle$ and $\operatorname{Im}(\bar{\tau})=\left\langle v_{1}, \ldots, v_{q-1}\right\rangle$ for all $q \leq p$. Using that $\bar{\sigma}=(\bar{\tau})^{p-1}$ we get:

$$
\operatorname{ker} \bar{\sigma}=\left\{\begin{array}{ll}
N_{q} & \text { if } q<p, \\
\left\langle v_{1}, \ldots, v_{p-1}\right\rangle & \text { if } q=p,
\end{array} \quad \operatorname{Im}(\bar{\sigma})= \begin{cases}0 & \text { if } q<p \\
\left\langle v_{1}\right\rangle & \text { if } q=p .\end{cases}\right.
$$

If $p=q$ the result is clear. If $q<p$ it follows from:

$$
\frac{\operatorname{ker}(\bar{\tau})}{\operatorname{Im}(\bar{\sigma})} \cong\left\langle v_{1}\right\rangle, \quad \frac{\operatorname{ker}(\bar{\sigma})}{\operatorname{Im}(\bar{\tau})} \cong\left\langle v_{q}\right\rangle
$$

Recall (see [11, Ch. V]) that the cohomology cross-product:

$$
H^{r}\left(G ; \mathbb{F}_{p}\right) \otimes_{\mathbb{Z}} H^{s}(G ; M) \longrightarrow H^{r+s}\left(G \times G ; \mathbb{F}_{p} \otimes_{\mathbb{Z}} M\right)
$$

followed by a diagonal approximation:

$$
H^{r+s}\left(G \times G ; \mathbb{F}_{p} \otimes_{\mathbb{Z}} M\right) \stackrel{\Delta^{*}}{\longrightarrow} H^{r+s}\left(G ; \mathbb{F}_{p} \otimes_{\mathbb{Z}} M\right) \cong H^{r+s}(G ; M)
$$

defines a cup-product and a graded $H^{*}\left(G ; \mathbb{F}_{p}\right)$-module structure on $H^{*}(G ; M)$, where $\mathbb{F}_{p} \otimes_{\mathbb{Z}} M$ is considered as a $G$-module for the diagonal action (and is isomorphic to $M$ as a $G$-module since $G$ acts trivially on $\mathbb{F}_{p}$ ). Here the diagonal approximation $\Delta^{*}$ is induced by the maps $\Delta_{r, s}: F_{r+s} \longrightarrow F_{r} \otimes F_{s}$ given by:

$$
\Delta_{r, s}(1)= \begin{cases}1 \otimes 1 & \text { for } r \text { even } \\ 1 \otimes g & \text { for } r \text { odd, } s \text { even } \\ \sum_{0 \leq i<j \leq p-1} g^{i} \otimes g^{j} & \text { for } r \text { odd, } s \text { odd }\end{cases}
$$

Let $\alpha \in H^{r}\left(G ; \mathbb{F}_{p}\right)$ and $\beta \in H^{s}(G ; M)$. Using again the natural identifications $\operatorname{Hom}_{G}\left(F_{r}, \mathbb{F}_{p}\right) \cong \mathbb{F}_{p}$ and $\operatorname{Hom}_{G}\left(F_{s}, M\right) \cong M$ one computes easily the cup-product $\alpha \cup \beta$ as follows:

(i) If $r$ is even, $\alpha \cup \beta=\alpha \beta$.

(ii) If $r$ is odd and $s$ is even, one has $\bar{\tau}(\beta)=0$ (see the proof of Lemma 3.1) so $g \beta=\beta$ and $\alpha \cup \beta=\alpha \beta$.

(iii) If $r$ is odd and $s$ is odd,

$$
\alpha \cup \beta=\alpha \cdot\left(\left(g+2 g^{2}+\cdots+(p-1) g^{p-1}\right) \beta\right) .
$$

We study the action of $g+2 g^{2}+\cdots+(p-1) g^{p-1}$ on $N_{q}$ for $1 \leq q \leq p$.

Lemma 3.2. As an endomorphism of $N_{q}$, with $1 \leq q \leq p$, one has:

$$
g+2 g^{2}+\cdots+(p-1) g^{p-1}= \begin{cases}0 & \text { if } q \leq p-2 \\ -\bar{\tau}^{q-1} & \text { if } q=p-1 \\ -\bar{\tau}^{q-1}-\bar{\tau}^{q-2} & \text { if } q=p .\end{cases}
$$


Proof. One computes:

$$
\begin{aligned}
\sum_{i=1}^{p-1} i g^{i} & =\sum_{i=1}^{p-1} \sum_{j=0}^{i} i\left(\begin{array}{l}
i \\
j
\end{array}\right) \bar{\tau}^{j}=\sum_{j=0}^{p-1}\left(\sum_{i=j}^{p-1} i\left(\begin{array}{l}
i \\
j
\end{array}\right)\right) \bar{\tau}^{j}=\sum_{j=0}^{p-1}\left(\sum_{k=0}^{p-1-j}(j+k)\left(\begin{array}{c}
j \\
j+k
\end{array}\right)\right) \bar{\tau}^{j} \\
& =\sum_{j=0}^{p-1}\left(j\left(\begin{array}{c}
p \\
j+1
\end{array}\right)+(j+1)\left(\begin{array}{c}
p \\
j+2
\end{array}\right)\right) \bar{\tau}^{j}
\end{aligned}
$$

where the last equality follows from an easy induction on $p$ (for any integer $p$ ). By reduction modulo $p$, all binomial coefficients $\left(\begin{array}{l}p \\ \ell\end{array}\right)$ vanish for $1 \leq \ell \leq p-1$ so:

$$
\sum_{i=1}^{p-1} i g^{i}=-\bar{\tau}^{p-1}-\bar{\tau}^{p-2} .
$$

Since $\bar{\tau}^{q}=0$ on $N_{q}$, the result follows.

In the special case $M=\mathbb{F}_{p}$, in case (iii) one obtains $\alpha \cup \beta=\alpha \beta$ if $p=2$ and $\alpha \cup \beta=0$ if $p \geq 3$. It follows that, as a graded commutative algebra:

$$
H^{*}\left(G ; \mathbb{F}_{p}\right) \cong \begin{cases}\mathbb{F}_{p}[u] & \text { if } p=2, \\ \Lambda^{*}(s) \otimes_{\mathbb{F}_{p}} \mathbb{F}_{p}[t] & \text { if } p \geq 3,\end{cases}
$$

where $\operatorname{deg}(u)=1, \operatorname{deg}(s)=1, \operatorname{deg}(t)=2$ and $\Lambda^{*}(s)$ denotes the exterior algebra over $\mathbb{F}_{p}$ generated by $s$ (see [1, Proposition 1.4.2]).

Proposition 3.3. $H^{*}\left(G ; N_{p}\right) \cong N_{p}^{G} \cong \mathbb{F}_{p}$ is a trivial $H^{*}\left(G ; \mathbb{F}_{p}\right)$-module. For $q<p$, $H^{*}\left(G ; N_{q}\right)$ is a free $H^{*}\left(G ; \mathbb{F}_{p}\right)$-module generated by $H^{0}\left(G ; N_{q}\right) \cong \mathbb{F}_{p}$.

Proof. This follows from Lemma 3.1 and the discussion above. The cases $q=p$ or $p=2$ are clear. In the case $p \geq 3$ and $q<p$, for $\alpha \in H^{r}\left(G ; \mathbb{F}_{p}\right)$ and $\beta \in H^{s}(G ; M)$ with $r$ odd and $s$ odd, following the notation used in the proof of Lemma $3.1 \beta$ can be represented by a class $v_{q}$ with $\bar{\sigma} v_{q}=0$. Since $\bar{\sigma}=\bar{\tau}^{p-1}$, using Lemma 3.2 one gets $\alpha \cup \beta=0$ in case (iii). The result follows.

We denote by $R$ the polynomial part of $H^{*}\left(G ; \mathbb{F}_{p}\right)$ (that is: $R=\mathbb{F}_{p}[u]$ for $p=2$ and $R=\mathbb{F}_{p}[t]$ for $p \geq 3$ ). We consider $\mathbb{F}_{p}$ as a $R$-module by evaluating at zero (setting $u=0$ for $p=2$ and $t=0$ for $p \geq 3$ ). For any $1 \leq q \leq p$, we consider $H^{*}\left(G ; N_{q}\right)$ as a $R$-module by the inclusion $R \hookrightarrow H^{*}\left(G ; \mathbb{F}_{p}\right)$.

\section{Corollary 3.4.}

(1) For $p=2$ and $q<p$, one has $\operatorname{dim}_{\mathbb{F}_{p}} \operatorname{Tor}_{0}^{R}\left(H^{*}\left(G ; N_{q}\right), \mathbb{F}_{p}\right)=1$ and for $i>0, \operatorname{Tor}_{i}^{R}\left(H^{*}\left(G ; N_{q}\right), \mathbb{F}_{p}\right)=0$.

(2) For $p \geq 3$ and $q<p$, one has $\operatorname{dim}_{\mathbb{F}_{p}} \operatorname{Tor}_{0}^{R}\left(H^{*}\left(G ; N_{q}\right), \mathbb{F}_{p}\right)=2$ and for $i>0, \operatorname{Tor}_{i}^{R}\left(H^{*}\left(G ; N_{q}\right), \mathbb{F}_{p}\right)=0$.

(3) For $p \geq 2$, one has:

$\operatorname{dim}_{\mathbb{F}_{p}} \operatorname{Tor}_{0}^{R}\left(H^{*}\left(G ; N_{p}\right), \mathbb{F}_{p}\right)=1=\operatorname{dim}_{\mathbb{F}_{p}} \operatorname{Tor}_{1}^{R}\left(H^{*}\left(G ; N_{p}\right), \mathbb{F}_{p}\right)=1$,

and for $i \geq 2, \operatorname{Tor}_{i}^{R}\left(H^{*}\left(G ; N_{p}\right), \mathbb{F}_{p}\right)=0$.

Proof. There is a length 2 projective resolution of $\mathbb{F}_{p}$ as a $R$-module given by:

$$
0 \longrightarrow R \stackrel{\phi}{\longrightarrow} R \longrightarrow \mathbb{F}_{p} \longrightarrow 0
$$


where $\phi: R \rightarrow R$ is the multiplication by $u$ for $p=2$ and by $t$ for $p \geq 3$, so $\operatorname{Tor}_{i}^{R}\left(H^{*}\left(G ; N_{q}\right), \mathbb{F}_{p}\right)=0$ for $i \geq 2$ and $q \leq p$.

(a) Assume that $q<p$. By Proposition [3.3. $H^{*}\left(G ; N_{q}\right)$ is a free $R$-module so $\operatorname{Tor}_{i}^{R}\left(H^{*}\left(G ; N_{q}\right), \mathbb{F}_{p}\right)=\{0\}$ for $i \geq 1$. Recall that:

$$
\operatorname{Tor}_{0}^{R}\left(H^{*}\left(G ; N_{q}\right), \mathbb{F}_{p}\right) \cong H^{*}\left(G ; N_{q}\right) \otimes_{R} \mathbb{F}_{p}
$$

For $p=2, H^{*}\left(G ; N_{q}\right)$ is generated by any non zero element $v \in H^{0}\left(G ; N_{q}\right)$ as a $R$-module so $\operatorname{dim}_{\mathbb{F}_{p}} H^{*}\left(G ; N_{q}\right) \otimes_{R} \mathbb{F}_{p}=1$; for $p \geq 3, H^{*}\left(G ; N_{q}\right)$ is again generated by any non zero $v \in H^{*}\left(G ; N_{q}\right)$ as a $H^{*}\left(G ; \mathbb{F}_{p}\right)$-module, so is generated by $v$ and $s v$ as a $R$-module: this gives $\operatorname{dim}_{\mathbb{F}_{p}} H^{*}\left(G ; N_{q}\right) \otimes_{R} \mathbb{F}_{p}=2$.

(b) Take $q=p$. From the length 2 resolution of $\mathbb{F}_{p}$ as a $R$-module, using Proposition 3.3 one gets:

$$
\operatorname{Tor}_{1}^{R}\left(H^{*}\left(G ; N_{p}\right), \mathbb{F}_{p}\right) \cong \operatorname{ker}\left(\phi: H^{*}\left(G ; N_{p}\right) \rightarrow H^{*}\left(G ; N_{p}\right)\right)=H^{*}\left(G ; N_{p}\right) .
$$

By Lemma 3.1 this space is one-dimensional so:

$$
\operatorname{Tor}_{0}^{R}\left(H^{*}\left(G ; N_{p}\right), \mathbb{F}_{p}\right) \cong H^{*}\left(G ; N_{p}\right) \otimes_{R} \mathbb{F}_{p} \cong \mathbb{F}_{p}
$$

\section{Equivariant COHOMOLOGY}

4.1. Basic facts on equivariant cohomology. Let $E G \rightarrow B G$ be a universal $G$-bundle in the category of CW-complexes. Denote by $X_{G}:=E G \times_{G} X$ the orbit space for the diagonal action of $G$ on the product $E G \times X$ and $f: X_{G} \rightarrow B G$ the map induced by the projection onto the first factor. The map $f$ is a locally trivial fibre bundle with typical fibre $X$ and structure group $G$. The equivariant cohomology of the pair $(X, G)$ with coefficients in $\mathbb{F}_{p}$ is defined by $H_{G}^{*}\left(X, \mathbb{F}_{p}\right):=H^{*}\left(X_{G}, \mathbb{F}_{p}\right)$, naturally endowed with a graded $H^{*}\left(B G, \mathbb{F}_{p}\right)$-module structure. Note that there is an isomorphism of graded algebras $H^{*}\left(B G, \mathbb{F}_{p}\right) \cong H^{*}\left(G ; \mathbb{F}_{p}\right)$. The Leray-Serre spectral sequence associated to the map $f$ gives a spectral sequence converging to the equivariant cohomology with coefficients in $\mathbb{F}_{p}$ :

$$
E_{2}^{r, s}:=H^{r}\left(G ; H^{s}\left(X, \mathbb{F}_{p}\right)\right) \Longrightarrow H_{G}^{r+s}\left(X, \mathbb{F}_{p}\right) .
$$

Remark 4.1. By assumption $X$ has the homotopy type of a finite $G$-CW-complex. Denote by $C^{*}(X)$ the cellular cochain complex of $X$ with coefficients in $\mathbb{F}_{p}$. The spaces $C^{s}(X)$ are finitely dimensional $\mathbb{F}_{p}$-vector spaces. Recall that $\varepsilon: F_{*} \rightarrow \mathbb{Z}$ denotes the projective resolution of $\mathbb{Z}$ as a trivial $\mathbb{Z}[G]$-module, and define the double complex $\beta_{G}^{r, s}(X):=\operatorname{Hom}_{G}\left(F_{r}, C^{s}(X)\right)$. As $C^{*}(X)$ is quasi-isomorphic to $R \Gamma\left(X, \mathbb{F}_{p}\right)$ in the derived category of $G$-modules, the cohomology of the total complex, the cohomology of the total complex $\operatorname{Tot} \beta_{G}(X)$ computes the equivariant cohomology (see Allday-Puppe [1, Theorem 1.2.8]): $H_{G}^{*}\left(X, \mathbb{F}_{p}\right) \cong H^{*}\left(\operatorname{Tot} \beta_{G}(X)\right)$. This yields a concrete description of the first quadrant spectral sequence converging to the equivariant cohomology.

4.2. Cohomology of the fixed locus. Recall that $R$ denotes the polynomial part of $H^{*}\left(G ; \mathbb{F}_{p}\right)$. We prove the following formula (see Allday-Puppe [1] for related results):

Proposition 4.2. For $p \geq 2$ one has:

$$
\begin{aligned}
& \qquad h^{*}\left(X^{G}, \mathbb{F}_{p}\right)=\nu \cdot\left(\operatorname{dim}_{\mathbb{F}_{p}} \operatorname{Tor}_{0}^{R}\left(H_{G}^{*}\left(X, \mathbb{F}_{p}\right), \mathbb{F}_{p}\right)-\operatorname{dim}_{\mathbb{F}_{p}} \operatorname{Tor}_{1}^{R}\left(H_{G}^{*}\left(X, \mathbb{F}_{p}\right), \mathbb{F}_{p}\right)\right) \\
& \text { with } \nu=1 \text { for } p=2 \text { and } \nu=\frac{1}{2} \text { for } p \geq 3 \text {. }
\end{aligned}
$$


Proof. The graded $R$-module $H_{G}^{*}\left(X, \mathbb{F}_{p}\right)$ is of finite type so it admits a minimal free resolution [1, Proposition A.4.12]:

$$
0 \longrightarrow L_{1} \longrightarrow L_{0} \longrightarrow H_{G}^{*}\left(X, \mathbb{F}_{p}\right) \longrightarrow 0
$$

such that $\operatorname{rank}_{R} L_{i}=\operatorname{dim}_{\mathbb{F}_{p}} \operatorname{Tor}_{i}^{R}\left(H_{G}^{*}\left(X, \mathbb{F}_{p}\right), \mathbb{F}_{p}\right)$. Write $R=\mathbb{F}_{p}[T]$ (with $T=u$ of degree one if $p=2$ and $T=t$ of degree two if $p \geq 3$ ). For $\alpha \in \mathbb{F}_{p}$, define $\mathbb{F}_{p, \alpha}:=R /(T-\alpha)$. This is consistent with the previous description $\mathbb{F}_{p} \cong \mathbb{F}_{p, 0}$ as an $R$-module. For $\alpha \neq 0$, the functor $-\otimes_{R} \mathbb{F}_{p, \alpha}$ is exact [1, Lemma A.7.2] so:

$$
\begin{aligned}
\operatorname{dim}_{\mathbb{F}_{p}} H_{G}^{*}\left(X, \mathbb{F}_{p}\right) \otimes_{R} \mathbb{F}_{p, \alpha}= & \operatorname{dim}_{\mathbb{F}_{p}} L_{0} \otimes_{R} \mathbb{F}_{p, \alpha}-\operatorname{dim}_{\mathbb{F}_{p}} L_{1} \otimes_{R} \mathbb{F}_{p, \alpha} \\
= & \operatorname{rank}_{R} L_{0}-\operatorname{rank}_{R} L_{1} \\
= & \operatorname{dim}_{\mathbb{F}_{p}} \operatorname{Tor}_{0}^{R}\left(H_{G}^{*}\left(X, \mathbb{F}_{p}\right), \mathbb{F}_{p}\right) \\
& \quad-\operatorname{dim}_{\mathbb{F}_{p}} \operatorname{Tor}_{1}^{R}\left(H_{G}^{*}\left(X, \mathbb{F}_{p}\right), \mathbb{F}_{p}\right) .
\end{aligned}
$$

For $\alpha \neq 0$, one has $H_{G}^{*}\left(X, \mathbb{F}_{p}\right) \otimes_{R} \mathbb{F}_{p, \alpha} \cong H^{*}\left(\beta_{G}(X) \otimes_{R} \mathbb{F}_{p, \alpha}\right)$ (this cohomology is computed with the total differential). We now use the following analogue of the localisation theorem in equivariant cohomology [1, Theorem 1.3.5, Theorem 1.4.5]: for $\alpha \neq 0$, one has

$$
H^{*}\left(\beta_{G}(X) \otimes_{R} \mathbb{F}_{p, \alpha}\right) \cong \begin{cases}H^{*}\left(X^{G}, \mathbb{F}_{p}\right) & \text { if } p=2 \\ H^{*}\left(X^{G}, \mathbb{F}_{p}\right) \otimes_{\mathbb{F}_{p}} \Lambda(s) & \text { if } p \geq 3\end{cases}
$$

The result follows.

If the spectral sequence of equivariant cohomology with coefficients in $\mathbb{F}_{p}$ degenerates at the $E_{2}$-term, it induces an isomorphism of graded $H^{*}\left(G ; \mathbb{F}_{p}\right)$-modules:

$$
H^{*}\left(G ; H^{*}\left(X, \mathbb{F}_{p}\right)\right) \cong H_{G}^{*}\left(X, \mathbb{F}_{p}\right)
$$

Using Corollary 3.4, Proposition 4.2 gives immediately:

Corollary 4.3. If the spectral sequence of equivariant cohomology with coefficients in $\mathbb{F}_{p}$ degenerates at the $E_{2}$-term, then for $p \geq 2$ one has:

$$
h^{*}\left(X^{G}, \mathbb{F}_{p}\right)=\sum_{1 \leq q<p} \ell_{q}^{*}(X)
$$

This formula can be stated differently, using only the parameter $\ell_{p}^{*}(X)$, that will appear to be the most important in the sequel:

Corollary 4.4. If the spectral sequence of equivariant cohomology with coefficients in $\mathbb{F}_{p}$ degenerates at the $E_{2}$-term, then for $p \geq 2$ one has:

$$
h^{*}\left(X^{G}, \mathbb{F}_{p}\right)=\operatorname{dim}_{\mathbb{F}_{p}} H^{*}\left(X, \mathbb{F}_{p}\right)^{G}-\ell_{p}^{*}(X) .
$$

Proof. Since each Jordan block of $H^{*}\left(X, \mathbb{F}_{p}\right)$ contains a one-dimensional invariant subspace, one gets $\operatorname{dim}_{\mathbb{F}_{p}} H^{*}\left(X, \mathbb{F}_{p}\right)^{G}=\sum_{1 \leq q \leq p} \ell_{q}^{*}(X)$. One conludes by using Corollary 4.3 . 
4.3. Degeneracy condition of the spectral sequence. Even under very nice conditions, one can not expect the collapsing of the spectral sequence in general. For instance, take $X$ a non-singular, real projective algebraic variety and $g: X(\mathbb{C}) \rightarrow X(\mathbb{C})$ the involution of complex conjugation, $G=\{1, g\}$ the order two group acting on $X(\mathbb{C})$. Then $X$ is called a $G M$-variety if the spectral sequence of equivariant cohomology with $E_{2}^{r, s}=H^{r}\left(G, H^{s}\left(X(\mathbb{C}), \mathbb{F}_{2}\right)\right)$ degenerates. See Krasnov [22, 23] for some examples of $G M$ and non- $G M$ varieties. In this section, we prove some degeneracy conditions that will be useful for certain symplectic holomorphic varieties.

Proposition 4.5. Assume that $\operatorname{dim}_{\mathbb{R}} X=4$ and $H^{\text {odd }}\left(X, \mathbb{F}_{p}\right)=0$. If $X$ has a fixed point for the action of $G$, then the spectral sequence of equivariant cohomology with coefficients in $\mathbb{F}_{p}$ degenerates at the $E_{2}$-term.

Proof. Let $x \in X$ be a fixed point for $G$. It induces a section $s: B G \rightarrow X_{G}$ of the projection $f: X_{G} \rightarrow B G$. Denote by

$$
u:=s_{*} 1 \in H^{4}\left(X_{G}, \mathbb{F}_{p}\right)
$$

the proper push-forward of the unit in $H^{*}\left(B G, \mathbb{F}_{p}\right)$. We can view $u$ as a morphism $u: \mathbb{F}_{p} \rightarrow \mathbb{F}_{p}[4]$ in the derived category of sheaves of $\mathbb{F}_{p}$-vector spaces over $X_{G}$. Pushing down yields a morphism

$$
q:=\mathbf{R} f_{*} u: \mathbf{R} f_{*} \mathbb{F}_{p} \rightarrow \mathbf{R} f_{*} \mathbb{F}_{p}[4]
$$

in the corresponding derived category of sheaves over $B G$. From Deligne [13, Proposition 2.1] modified by the arguments of [13, Remarque (1.9), $s=2$ ] (where we use the assumption $H^{\text {odd }}\left(X, \mathbb{F}_{p}\right)=0$ ) we get that if $q: R^{0} f_{*} \mathbb{F}_{p} \rightarrow R^{4} f_{*} \mathbb{F}_{p}$ is an isomorphism, then $\mathbb{F}_{p}$ satisfies the Lefschetz condition relative to $u$, that is:

$$
\mathbf{R} f_{*} \mathbb{F}_{p} \cong \bigoplus_{i} R^{i} f_{*} \mathbb{F}_{p}[-i]
$$

and the spectral sequence of equivariant cohomology with coefficients in $\mathbb{F}_{p}$ degenerates at the $E_{2}$-term.

In order to show that $q$ is an isomorphism, note that its source and target, being higher direct images of a constant sheaf along a locally trivial fibration, are locally constant sheaves. Thus it is enough to show that $q$ is an isomorphism fibrewise. This follows from base change, as the fibre of $R^{i} f_{*} \mathbb{F}_{p}$ at a point $t \in B G$ is just $H^{i}\left(X, \mathbb{F}_{p}\right)$ and the fibre of the morphism $q$ at $t$ is the multiplication by the fundamental class $[x] \in H^{4}\left(X, \mathbb{F}_{p}\right)$ of the fixed point $x$.

Let $\mathcal{F}$ be a vector bundle on $X$. Recall that a $G$-linearisation of $\mathcal{F}$ is given by the data of homomorphisms $\phi_{g}: g^{*} \mathcal{F} \rightarrow \mathcal{F}$ for all $g \in G$ such that the cocycle condition $\phi_{h} \circ h^{*}\left(\phi_{g}\right)=\phi_{h g}: g^{*} h^{*} \mathcal{F} \rightarrow \mathcal{F}$ is fulfilled for all $g, h \in G$. A $G$-linearised vector bundle is a vector bundle together with the data of a $G$-linearisation. Note that $G$ equivariant resolutions exist, see Elagin [15. Natural examples are the (co)tangent bundle on $X$ (where the $G$-linearization is given by pullback along the action of $G$ ) or the sheaf of section $\mathcal{O}(D)$ for any divisor $D$ on $X$ that is globally invariant for the action of $G$.

A $G$-linearisation on a vector bundle $\mathcal{F}$ induces an ordinary $G$-action on the étale space of $\mathcal{F}$, which we denote by $\mathcal{F}$ again, such that the natural projection $\mathcal{F} \rightarrow X$ becomes a $G$-equivariant map. We can then form the space $\mathcal{F}_{G}:=\mathcal{F} \times_{G} E G$, which has a natural map to $X_{G}$, making it canonically into a vector bundle over $X_{G}$. If 
we restrict $\mathcal{F}_{G}$ to a fibre of $f: X_{G} \rightarrow B G$ (all of which are isomorphic to $X$ ), it becomes the vector bundle $\mathcal{F}$ over $X$ again.

If $\mathcal{F}$ has the additional structure of a complex vector bundle and the $G$-linearisation of $\mathcal{F}$ is compatible with this structure, the induced bundle $\mathcal{F}_{G}$ inherits this structure as a complex vector bundle. Given two $G$-linearised (complex) vector bundles $\mathcal{F}$ and $\mathcal{F}^{\prime}$ over $X$, there is the obvious notion of a $G$-equivariant homomorphism between $\mathcal{F}$ and $\mathcal{F}^{\prime}$. It induces naturally an ordinary homomorphism between $\mathcal{F}_{G}$ and $\mathcal{F}_{G}^{\prime}$. This construction is compatible with the notion of exact sequences, so we get in fact a group homomorphism from the $G$-equivariant Grothendieck group $K_{G}^{0}(X)$ of $X$ to the ordinary Grothendieck group $K^{0}\left(X_{G}\right)$ of (complex) vector bundles. By forgetting the $G$-linearisations, one defines another group homomorphism from $K_{G}^{0}(X) \rightarrow K^{0}(X)$.

This allows one to construct classes in the equivariant cohomology. Let $\alpha$ be a characteristic class of complex $K$-theory with values in $\mathbb{F}_{p}$ (in the sequel, we will use reductions modulo $p$ of integral characteristic classes like integral linear combinations of Chern classes). Let $\mathcal{F}$ be a $G$-equivariant vector bundle, or more generally a class in the $G$-equivariant Grothendieck group $K_{G}^{0}(X)$. Then $\alpha\left(\mathcal{F}_{G}\right) \in$ $H^{*}\left(X_{G}, \mathbb{F}_{p}\right)$. By the naturality of characteristic classes, the restriction of $\alpha\left(\mathcal{F}_{G}\right)$ to a fibre of $f: X_{G} \rightarrow B G$ is just $\alpha(\mathcal{F})$.

Proposition 4.6. Assume that $\operatorname{dim}_{\mathbb{R}} X=8$ and $H^{\text {odd }}\left(X, \mathbb{F}_{p}\right)=0$. Let $\mathcal{F} \in K_{G}^{0}(X)$ be a class in the equivariant complex $K$-theory of $X$ and $c:=\alpha_{2}(\mathcal{F}) \in H^{4}\left(X, \mathbb{F}_{p}\right)$ a characteristic class. Assume that the multiplication maps

$$
\begin{aligned}
& H^{2}\left(X, \mathbb{F}_{p}\right) \rightarrow H^{6}\left(X, \mathbb{F}_{p}\right), \quad \beta \mapsto c \cup \beta \\
& H^{0}\left(X, \mathbb{F}_{p}\right) \rightarrow H^{8}\left(X, \mathbb{F}_{p}\right), \quad \beta \mapsto c^{2} \cup \beta
\end{aligned}
$$

are isomorphisms. Then the spectral sequence of equivariant cohomology with coefficients in $\mathbb{F}_{p}$ degenerates at the $E_{2}$-term.

Proof. The proof is virtually the same as for proposition 4.5. Denoting as above $u:=\alpha\left(\mathcal{F}_{G}\right) \in H^{4}\left(X_{G}, \mathbb{F}_{p}\right)$ and $q:=\mathbf{R} f_{*} u: \mathbf{R} f_{*} \mathbb{F}_{p} \rightarrow \mathbf{R} f_{*} \mathbb{F}_{p}[4]$, we use again Deligne [13. Proposition (2.1)] modified by the arguments of [13, Remarque (1.9), $s=2$ ]: if $q: R^{2} f_{*} \mathbb{F}_{p} \rightarrow R^{6} f_{*} \mathbb{F}_{p}$ and $q^{2}: R^{0} f_{*} \mathbb{F}_{p} \rightarrow R^{8} f_{*} \mathbb{F}_{p}$ are isomorphisms, then $\mathbb{F}_{p}$ satisfies the Lefschetz condition relative to $u$ and the spectral sequence degenerates at the $E_{2}$-term. Again this can be checked fibrewise, where these maps are the multiplications by $c$ and $c^{2}$ respectively.

Remark 4.7. As an example, assume that $X$ is a smooth complex algebraic variety of complex dimension two that possesses a $G$-fixed point $x$. The skyscraper sheaf to this point defines a class $[x]$ in $K_{G}^{0}(X)$ (after a finite $G$-equivariant resolution). For $\alpha$ take the second Chern class $c_{2}$. It follows that $u:=c_{2}([x]) \in H^{4}\left(X_{G}, \mathbb{F}_{p}\right)$ is a class whose restriction to each fibre $X$ of $p: X_{G} \rightarrow B G$ is just the fundamental class of the point $x$ since $\operatorname{dim}_{\mathbb{R}} X=4$. This is the class used in Proposition 4.5.

Remark 4.8. The preceding two propositions are valid for any finite group $G$ acting on $X$, not only $\mathbb{Z} / p \mathbb{Z}$.

\section{TWO INTEGRAL PARAMETERS}

Assume that $H^{*}(X, \mathbb{Z})$ is torsion-free. By the universal coefficient theorem, one has $H^{*}\left(X, \mathbb{F}_{p}\right) \cong H^{*}(X, \mathbb{Z}) \otimes_{\mathbb{Z}} \mathbb{F}_{p}$ and the homomorphisms of reduction modulo $p$, 
denoted by

$$
\kappa_{k}: H^{k}(X, \mathbb{Z}) \longrightarrow H^{k}\left(X, \mathbb{F}_{p}\right)
$$

are surjective for all $k$.

Let $\xi_{p}$ be a primitive $p$-th root of the unity, $K:=\mathbb{Q}\left(\xi_{p}\right)$ and $\mathcal{O}_{K}:=\mathbb{Z}\left[\xi_{p}\right]$ the ring of algebraic integers of $K$. By a classical theorem of Masley-Montgomery [30, $\mathcal{O}_{K}$ is a PID if and only if $p \leq 19$. The $G$-module structure of $\mathcal{O}_{K}$ is defined by $g \cdot x=\xi_{p} x$ for $x \in \mathcal{O}_{K}$. For any $a \in \mathcal{O}_{K}$, we denote by $\left(\mathcal{O}_{K}, a\right)$ the module $\mathcal{O}_{K} \oplus \mathbb{Z}$ whose $G$-module structure is defined by $g \cdot(x, k)=\left(\xi_{p} x+k a, k\right)$.

Proposition 5.1. Assume that $H^{*}(X, \mathbb{Z})$ is torsion-free and $3 \leq p \leq 19$. Then for $0 \leq k \leq \operatorname{dim}_{\mathbb{R}} X$ one has:

(1) $\ell_{i}^{k}(X)=0$ for $2 \leq i \leq p-2$.

(2) $\operatorname{rank}_{\mathbb{Z}} H^{k}(X, \mathbb{Z})=p \ell_{p}^{k}(X)+(p-1) \ell_{p-1}^{k}(X)+\ell_{1}^{k}(X)$.

(3) $\operatorname{dim}_{\mathbb{F}_{p}} H^{k}\left(X, \mathbb{F}_{p}\right)^{G}=\ell_{p}^{k}(X)+\ell_{p-1}^{k}(X)+\ell_{1}^{k}(X)$.

(4) $\operatorname{rank}_{\mathbb{Z}} H^{k}(X, \mathbb{Z})^{G}=\ell_{p}^{k}(X)+\ell_{1}^{k}(X)$.

Proof. By a theorem of Diederichsen and Reiner [12, Theorem 74.3], $H^{k}(X, \mathbb{Z})$ is isomorphic as a $\mathbb{Z}[G]$-module to a direct sum:

$$
\left(A_{1}, a_{1}\right) \oplus \cdots \oplus\left(A_{r}, a_{r}\right) \oplus A_{r+1} \oplus \cdots \oplus A_{r+s} \oplus Y
$$

where the $A_{i}$ are fractional ideals in $K, a_{i} \in A_{i}$ are such that $a_{i} \notin\left(\xi_{p}-1\right) A_{i}$ and $Y$ is a free $\mathbb{Z}$-module of finite rank on which $G$ acts trivially. The $G$-module structure on $A_{i}$ is defined by $g \cdot x=\xi_{p} x$ for all $x \in A_{i}$, and $\left(A_{i}, a_{i}\right)$ denotes the module $A_{i} \oplus \mathbb{Z}$ whose $G$-module structure is defined by $g \cdot(x, k)=\left(\xi_{p} x+k a_{i}, k\right)$. Since $\mathcal{O}_{K}$ is a PID, there is only one ideal class in $K$ so we have an isomorphism of $\mathbb{Z}[G]$-modules:

$$
H^{k}(X, \mathbb{Z}) \cong \oplus_{i=1}^{r}\left(\mathcal{O}_{K}, a_{i}\right) \oplus \mathcal{O}_{K}^{\oplus s} \oplus \mathbb{Z}^{\oplus t}
$$

for some $a_{i} \notin\left(\xi_{p}-1\right) \mathcal{O}_{K}$. The matrix of the action of $g$ acting on $\mathcal{O}_{K}$ is:

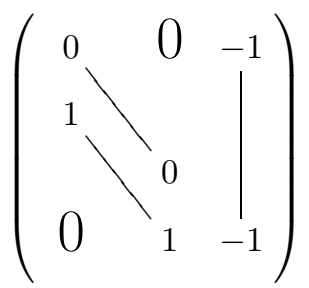

so its minimal polynomial over $\mathbb{Q}$ is the cyclotomic polynomial $\Phi_{p}$, hence $\mathcal{O}_{K}$ has no $G$-invariant element over $\mathbb{Z}$. Over $\mathbb{F}_{p}$, the minimal polynomial of $\mathcal{O}_{K} \otimes_{\mathbb{Z}} \mathbb{F}_{p}$ is $\Phi_{p}(X)=(X-1)^{p-1}$, so $\mathcal{O}_{K} \otimes_{\mathbb{Z}} \mathbb{F}_{p}$ is isomorphic to $N_{p-1}$ as a $\mathbb{F}_{p}[G]$-module. The matrix of the action of $g$ on $\left(\mathcal{O}_{K}, a\right)$ is:

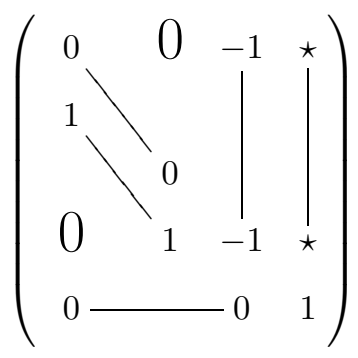


so its minimal polynomial over $\mathbb{Q}$ is $(X-1) \Phi_{p}(X)=X^{p}-1$, hence the subspace of invariants $\left(\mathcal{O}_{K}, a\right)^{G}$ is one-dimensional. Over $\mathbb{F}_{p}$, the minimal polynomial of $\left(\mathcal{O}_{K}, a\right) \otimes_{\mathbb{Z}} \mathbb{F}_{p}$ is $(X-1)^{p}$, so $\left(\mathcal{O}_{K}, a\right) \otimes_{\mathbb{Z}} \mathbb{F}_{p}$ is isomorphic to $N_{p} \cong \mathbb{F}_{p}[G]$ as a $\mathbb{F}_{p}[G]$-module. By reduction modulo $p$, the universal coefficient theorem implies:

$$
H^{k}\left(X, \mathbb{F}_{p}\right) \cong N_{p}^{\oplus r} \oplus N_{p-1}^{\oplus s} \oplus N_{1}^{\oplus t}
$$

as $\mathbb{F}_{p}[G]$-modules, so $\ell_{p}^{k}(X)=r, \ell_{p-1}^{k}(X)=s, \ell_{1}^{k}(X)=t$ and $\ell_{i}^{k}(X)=0$ for $2 \leq i \leq p-2$, this proves (11) and (2). Since each block contains a one-dimensional $G$-invariant subspace, this implies also that:

$$
\operatorname{dim}_{\mathbb{F}_{p}} H^{k}\left(X, \mathbb{F}_{p}\right)^{G}=\ell_{p}^{k}(X)+\ell_{p-1}^{k}(X)+\ell_{1}^{k}(X),
$$

this proves (3). Over $\mathbb{Z}$, only the trivial $G$-module in $H^{k}(X ; \mathbb{Z})$ and the $G$-modules $\left(\mathcal{O}_{K}, a\right)$ contain a $G$-invariant subspace, of dimension 1 , so:

$$
\operatorname{rank}_{\mathbb{Z}} H^{k}(X, \mathbb{Z})^{G}=r+t=\ell_{p}^{k}(X)+\ell_{1}^{k}(X),
$$

this proves (41).

Remark 5.2. Assume that $H^{*}(X, \mathbb{Z})$ is torsion-free and $p=2$. The above argument is much more basic and one gets easily, with the same notation, that $\ell_{1}^{k}(X)=s+t$, $\ell_{2}^{k}(X)=r, \operatorname{rank}_{\mathbb{Z}} H^{k}(X, \mathbb{Z})^{G}=r+t, \operatorname{dim}_{\mathbb{F}_{2}} H^{k}\left(X, \mathbb{F}_{2}\right)^{G}=\ell_{1}^{k}(X)+\ell_{2}^{k}(X)$.

Recall that $G=\langle g\rangle, \tau=g-1 \in \mathbb{Z}[G]$ and $\sigma=1+g+\cdots+g^{p-1} \in \mathbb{Z}[G]$. We denote also by $g, \tau, \sigma$ their actions on any $\mathbb{Z}[G]$-module. For $1 \leq k \leq \operatorname{dim}_{\mathbb{R}} X-1$ we define:

$$
\begin{aligned}
& \mathrm{T}_{G}^{k}(X):=\operatorname{ker}(\tau) \cap H^{k}(X, \mathbb{Z}), \\
& \mathrm{S}_{G}^{k}(X):=\operatorname{ker}(\sigma) \cap H^{k}(X, \mathbb{Z}) .
\end{aligned}
$$

As kernels these modules are primitive in $H^{k}(X, \mathbb{Z})$.

Lemma 5.3. Assume that $H^{*}(X, \mathbb{Z})$ is torsion-free and $2 \leq p \leq 19$. Then for all $k, \frac{H^{k}(X, \mathbb{Z})}{\mathrm{T}_{G}^{k}(X) \oplus \mathrm{S}_{G}^{k}(X)}$ is a p-torsion module.

Proof. First observe that $\mathrm{T}_{G}^{k}(X) \cap \mathrm{S}_{G}^{k}(X)=\{0\}$ since $H^{k}(X, \mathbb{Z})$ has no $p$-torsion. As in the proof of Proposition 5.1 for each $k$ one has a $\mathbb{Z}[G]$-module decomposition:

$$
H^{k}(X, \mathbb{Z}) \cong \oplus_{i=1}^{r}\left(\mathcal{O}_{K}, a_{i}\right) \oplus \mathcal{O}_{K}^{\oplus s} \oplus \mathbb{Z}^{\oplus t} .
$$

It is clear that $\mathbb{Z}^{\oplus t} \subset \mathrm{T}_{G}^{k}(X)$ and $\mathcal{O}_{K}^{\oplus s} \subset \mathrm{S}_{G}^{k}(X)$. In any term $\left(\mathcal{O}_{K}, a\right)=\mathcal{O}_{K} \oplus \mathbb{Z}$, denoting $v:=(0,1)$ in this decomposition, we show that $p v \in \mathrm{T}_{G}^{k}(X) \oplus \mathrm{S}_{G}^{k}(X)$. For this, observe that the quotient of $\mathcal{O}_{K}$ by its maximal ideal $\left(\xi_{p}-1\right)$ is $\mathbb{Z} / p \mathbb{Z}$, so for any $x \in \mathcal{O}_{K}$ there exists $z \in \mathcal{O}_{K}$ such that $p x=\left(\xi_{p}-1\right) z$. One has $\tau(v)=(a, 0)$ hence there exists $z \in \mathcal{O}_{K}$ such that $\tau(p v)=(p a, 0)=\left(\left(\xi_{p}-1\right) z, 0\right)$. Now $\tau((z, 0))=\left(\left(\xi_{p}-1\right) z, 0\right)$ hence $\tau(p v-(z, 0))=0$ and $\sigma(z, 0)=0$ so finally

$$
p v=(p v-(z, 0))+(z, 0) \in \mathrm{T}_{G}(X) \oplus \mathrm{S}_{G}(X) .
$$

This shows that $\frac{H^{k}(X, \mathbb{Z})}{\mathrm{T}_{G}^{k}(X) \oplus \mathrm{S}_{G}^{k}(X)}$ is a torsion module, and that it has only $p$-torsion.

Remark 5.4. As a consequence of the proof of Lemma 5.3. observe that $\frac{H^{k}(X, \mathbb{Z})}{\mathrm{T}_{G}^{k}(X) \oplus \mathrm{S}_{G}^{k}(X)}$ is a trivial $G$-module: it is generated by the vectors $v=(0,1)$ of each factor $\left(\mathcal{O}_{K}, a\right)$ appearing in the decomposition above, and the action of $G$ is

$$
g \cdot v=a+v \equiv v \quad \bmod \mathrm{T}_{G}^{k}(X) \oplus \mathrm{S}_{G}^{k}(X)
$$


Definition 5.5. Assume that $H^{*}(X, \mathbb{Z})$ is torsion-free and $2 \leq p \leq 19$. For $1 \leq$ $k \leq \operatorname{dim}_{\mathbb{R}} X-1$ we define $\mathrm{a}_{G}^{k}(X) \in \mathbb{N}$ such that:

$$
\frac{H^{k}(X, \mathbb{Z})}{\mathrm{T}_{G}^{k}(X) \oplus \mathrm{S}_{G}^{k}(X)} \cong\left(\frac{\mathbb{Z}}{p \mathbb{Z}}\right)^{\mathrm{a}_{G}^{k}(X)}
$$

Lemma 5.6. Assume that $H^{*}(X, \mathbb{Z})$ is torsion-free. For $1 \leq k \leq \operatorname{dim}_{\mathbb{R}} X-1$, one has:

$$
\kappa_{k}\left(\mathrm{~T}_{G}^{k}(X) \oplus \mathrm{S}_{G}^{k}(X)\right)=\operatorname{ker}(\bar{\sigma}) \cap H^{k}\left(X, \mathbb{F}_{p}\right) .
$$

Proof. It is clear that $\kappa_{k}\left(\mathrm{~S}_{G}^{k}(X)\right) \subset \operatorname{ker}(\bar{\sigma})$. Similarly, since $\mathrm{T}_{G}^{k}(X)=\operatorname{ker}(\tau)$ and $\bar{\sigma}=\bar{\tau}^{p-1}$, one has $\kappa_{k}\left(\mathrm{~T}_{G}^{k}(X)\right) \subset \operatorname{ker}(\bar{\sigma})$. Take $x \in H^{k}(X, \mathbb{Z})$ such that $\kappa(x) \in$ $\operatorname{ker}(\bar{\sigma})$. By Lemma 5.3 one can write $p x=u+v$ for some $u \in \mathrm{T}_{G}^{k}(X), v \in \mathrm{S}_{G}^{k}(X)$. Now $0=\bar{\sigma} \kappa(x)=\kappa \sigma(x)$ and $\sigma(x)=u$. This shows that $u=p u^{\prime}$ for some $u^{\prime} \in \mathrm{T}_{G}^{k}(X)$. Hence $p x=p u^{\prime}+v$, giving $v=p v^{\prime}$ for some $v^{\prime} \in \mathrm{S}_{G}^{k}(X)$, so finally $x \in \mathrm{T}_{G}^{k}(X) \oplus \mathrm{S}_{G}^{k}(X)$.

Corollary 5.7. Assume that $H^{*}(X, \mathbb{Z})$ is torsion-free. For $1 \leq k \leq \operatorname{dim}_{\mathbb{R}} X-1$ there is an isomorphism of $\mathbb{F}_{p}$-vector spaces:

$$
\frac{H^{k}(X, \mathbb{Z})}{\mathrm{T}_{G}^{k}(X) \oplus \mathrm{S}_{G}^{k}(X)} \cong \frac{H^{k}\left(X, \mathbb{F}_{p}\right)}{\operatorname{ker}(\bar{\sigma}) \cap H^{k}\left(X, \mathbb{F}_{p}\right)}
$$

Corollary 5.8. Assume that $H^{*}(X, \mathbb{Z})$ is torsion-free. For $1 \leq k \leq \operatorname{dim}_{\mathbb{R}} X-1$ and $2 \leq p \leq 19$ one has:

$$
\mathrm{a}_{G}^{k}(X)=\ell_{p}^{k}(X) \text {. }
$$

Proof. By Corollary 5.7 one has $\operatorname{dim}_{\mathbb{F}_{p}} \operatorname{ker}\left(\bar{\sigma}_{\mid H^{k}\left(X, \mathbb{F}_{p}\right)}\right)=h^{k}\left(X, \mathbb{F}_{p}\right)-\mathrm{a}_{G}^{k}(X)$ and by Lemma 3.1 and its proof, $\operatorname{dim}_{\mathbb{F}_{p}} \operatorname{ker}\left(\bar{\sigma}_{\mid H^{k}\left(X, \mathbb{F}_{p}\right)}\right)=h^{k}\left(X, \mathbb{F}_{p}\right)-\ell_{p}^{k}(X)$. The result follows.

Assume now that $3 \leq p \leq 19$. There is an exact sequence:

$$
0 \longrightarrow(\sigma) \longrightarrow \mathbb{Z}[G] \longrightarrow \mathbb{Z}\left[\xi_{p}\right] \longrightarrow 0
$$

given by $g \mapsto \xi_{p}$. Since the $p$-th cyclotomic polynomial $\Phi_{p}(X) \in \mathbb{Q}[X]$ is irreducible and $\sigma=\Phi_{p}(g)$, one deduces that $\mathrm{S}_{G}^{k}(X)$ is a free $\mathcal{O}_{K}$-module. Since $\mathcal{O}_{K}$ is a free $\mathbb{Z}$-module of rank $p-1$, we introduce the following definition:

Definition 5.9. Assume that $2 \leq p \leq 19$. For $1 \leq k \leq \operatorname{dim}_{\mathbb{R}} X-1$ we define $\mathrm{m}_{G}^{k}(X) \in \mathbb{N}$ such that:

$$
\operatorname{rank}_{\mathbb{Z}} \mathrm{S}_{G}^{k}(X)=\mathrm{m}_{G}^{k}(X)(p-1) .
$$

Corollary 5.10. Assume that $H^{*}(X, \mathbb{Z})$ is torsion-free. For $1 \leq k \leq \operatorname{dim}_{\mathbb{R}} X-1$ and $3 \leq p \leq 19$ one has:

$$
\mathrm{m}_{G}^{k}(X)=\ell_{p}^{k}(X)+\ell_{p-1}^{k}(X) .
$$

Proof. By Proposition 5.1(4) one has:

$$
\operatorname{rank}_{\mathbb{Z}} \mathrm{T}_{G}^{k}(X)=\ell_{1}^{k}(X)+\ell_{p}^{k}(X)
$$

and by Lemma $5.3, \operatorname{rank}_{\mathbb{Z}} \mathrm{T}_{G}^{k}(X)+\operatorname{rank}_{\mathbb{Z}} \mathrm{S}_{G}^{k}(X)=h^{k}(X, \mathbb{Z})=h^{k}\left(X, \mathbb{F}_{p}\right)$ one gets:

$$
h^{k}\left(X, \mathbb{F}_{p}\right)=\ell_{1}^{k}(X)+\ell_{p}^{k}(X)+\mathrm{m}_{G}^{k}(X)(p-1) .
$$

Since $h^{k}\left(X, \mathbb{F}_{p}\right)=p \ell_{p}^{k}(X)+(p-1) \ell_{p-1}^{k}(X)+\ell_{1}^{k}(X)$, one gets the result. 
As a consequence of Corollary 4.3 and Proposition 5.1 using Corollaries $5.8 \& 5.10$ we get the following explicit relation between the cohomology of the fixed locus and the parameters $\mathrm{a}_{G}, \mathrm{~m}_{G}$.

Corollary 5.11. Assume that $p=2, H^{*}(X, \mathbb{Z})$ is torsion-free and the spectral sequence of equivariant cohomology with coefficients in $\mathbb{F}_{2}$ degenerates at the $E_{2}$ term. Then:

$$
h^{*}\left(X^{G}, \mathbb{F}_{2}\right)=h^{*}\left(X, \mathbb{F}_{2}\right)-2 \sum_{k=1}^{\operatorname{dim}_{\mathbb{R}} X-1} \mathrm{a}_{G}^{k}(X) .
$$

Corollary 5.12. Assume that $3 \leq p \leq 19, H^{*}(X, \mathbb{Z})$ is torsion-free and the spectral sequence of equivariant cohomology with coefficients in $\mathbb{F}_{p}$ degenerates at the $E_{2}$ term. Then:

$$
h^{*}\left(X^{G}, \mathbb{F}_{p}\right)=h^{*}\left(X, \mathbb{F}_{p}\right)-2 \sum_{k=1}^{\operatorname{dim}_{\mathbb{R}} X-1} \mathrm{a}_{G}^{k}(X)-(p-2) \sum_{k=1}^{\operatorname{dim}_{\mathbb{R}} X-1} \mathrm{~m}_{G}^{k}(X) .
$$

Proposition 5.13. The following inequalities hold for all $k$ :

$$
\begin{aligned}
& 0 \leq \mathrm{m}_{G}^{k}(X)(p-1) \leq h^{k}\left(X, \mathbb{F}_{p}\right), \\
& 0 \leq \mathrm{a}_{G}^{k}(X) \leq \min \left\{\mathrm{m}_{G}^{k}(X)(p-1), h^{k}\left(X, \mathbb{F}_{p}\right)-\mathrm{m}_{G}^{k}(X)(p-1)\right\} .
\end{aligned}
$$

Proof. One has $\frac{H^{k}(X, \mathbb{Z})}{\mathrm{T}_{G}^{k}(X) \oplus \mathrm{S}_{G}^{k}(X)} \cong\left(\frac{\mathbb{Z}}{p \mathbb{Z}}\right)^{\mathrm{a}_{G}^{k}(X)}$ : since $\mathrm{T}_{G}^{k}(X)$ and $\mathrm{S}_{G}^{k}(X)$ are primitive, the divisible classes are of the form $\frac{1}{p}(u+v)$ with $u \in \mathrm{T}_{G}^{k}(X)$ and $v \in \mathrm{S}_{G}^{k}(X)$. The integer $\mathrm{a}_{G}^{k}(X)$ is the maximal number of divisible classes independant modulo $\mathrm{T}_{G}^{k}(X) \oplus \mathrm{S}_{G}^{k}(X)$ and is thus smaller than $\operatorname{rank}\left(\mathrm{T}_{G}^{k}(X)\right)$ and $\operatorname{rank}\left(\mathrm{S}_{G}^{k}(X)\right)$.

\section{Automorphisms of IRREDUCIBLE HOLOMORPHIC SYMPLECTIC MANIFOLDS}

6.1. Basic facts on lattices. Let $(\Lambda,\langle\cdot, \cdot\rangle)$ be a lattice (a free $\mathbb{Z}$-module with an integral, bilinear symmetric, non-degenerate two-form). If $\Gamma \subset \Lambda$ is a sublattice, the dual lattice is by definition $\Gamma^{*}:=\operatorname{Hom}_{\mathbb{Z}}(\Gamma, \mathbb{Z})$. Recall that:

$$
\Gamma^{*} \cong\{x \in \Gamma \otimes \mathbb{Q} \mid\langle x, y\rangle \in \mathbb{Z} \text { for all } y \in \Gamma\} .
$$

Then $\Gamma \subset \Gamma^{*}$ is a sublattice of the same rank, so the quotient $A_{\Gamma}:=\Gamma^{*} / \Gamma$ is a finite abelian group, called the discriminant group. Its order is denoted by $\operatorname{disc}(\Gamma):=\left|A_{\Gamma}\right|$ and called the discriminant of $\Gamma$.

A lattice $\Lambda$ is called unimodular if $\Lambda^{*}=\Lambda$, that is $A_{\Lambda}=0$. In a basis $\left(e_{i}\right)_{i}$ of $\Lambda$, consider the matrix $M=\left(\left\langle e_{i}, e_{j}\right\rangle\right)_{i, j}$ : then $\operatorname{disc}(\Lambda)=\operatorname{det}(M)$.

A sublattice $\Gamma \subset \Lambda$ is called primitive if $\Lambda / \Gamma$ is a free $\mathbb{Z}$-module. If $\Lambda$ is unimodular and $\Gamma \subset \Lambda$ is primitive, then $A_{\Gamma} \cong A_{\Gamma^{\perp}}$.

Let $p$ be a prime number. A lattice $\Gamma$ is called p-elementary if $A_{\Gamma} \cong(\mathbb{Z} / p \mathbb{Z})^{a(\Gamma)}$ for some integer $a(\Gamma)$. In particular, $\operatorname{disc}(\Gamma)=p^{a(\Gamma)}$. If $\Gamma$ is primitively embedded in a unimodular lattice $\Lambda$, then $\Gamma^{\perp}$ is also $p$-elementary and $\operatorname{disc}(\Gamma)=\operatorname{disc}\left(\Gamma^{\perp}\right)$.

A lattice $\Gamma$ is called even if $(x, x) \equiv 0 \bmod 2$ for all $x \in \Gamma$. Equivalently, the diagonal elements of the matrix $M$ are even. 
6.2. Basic facts on irreducible holomorphic symplectic manifolds. A compact Kähler manifold $X$ is called irreducible symplectic if $X$ is simply connected and $H^{0}\left(X, \Omega_{X}^{2}\right)$ is spanned by an everywhere non-degenerate closed two-form, denoted $\omega_{X}$. We have a Hodge decomposition:

$$
H^{2}(X, \mathbb{C})=H^{2,0}(X) \oplus H^{1,1}(X) \oplus H^{0,2}(X)
$$

and we put $H^{1,1}(X)_{\mathbb{R}}:=H^{1,1}(X) \cap H^{2}(X, \mathbb{R})$. The second cohomology group $H^{2}(X, \mathbb{Z})$ is torsion-free and equipped with a bilinear symmetric, even, non-degenerate two-form of signature $\left(3, b_{2}(X)-3\right)$, called the Beauville-Bogomolov form [4, and such that - after scalar extension - $H^{1,1}(X)$ is orthogonal to $H^{2,0}(X) \oplus H^{0,2}(X)$. We denote by $\langle\cdot, \cdot\rangle$ the bilinear form and by $q$ the associated quadratic form. The Néron-Severi group of $X$ is defined by:

$$
\mathrm{NS}(X):=H^{1,1}(X)_{\mathbb{R}} \cap H^{2}(X, \mathbb{Z})=\left\{x \in H^{2}(X, \mathbb{Z}) \mid\left\langle x, \omega_{X}\right\rangle=0\right\} .
$$

We set $\rho(X):=\operatorname{rank}(\mathrm{NS}(X))$ the Picard number of $X$ and $\mathrm{T}(X):=\mathrm{NS}(X)^{\perp}$ the orthogonal complement of $\mathrm{NS}(X)$ in $H^{2}(X, \mathbb{Z})$ for the quadratic form, called the transcendental lattice. Note that $\operatorname{NS}(X)$ and $\mathrm{T}(X)$ are primitively embedded in $H^{2}(X, \mathbb{Z})$. We denote the signature of a lattice by $\left(n_{1}, n_{2}, n_{3}\right)$ where $n_{1}$ is the number of positive eigenvalues, $n_{2}$ of the zero eigenvalues and $n_{3}$ of the negative eigenvalues of the associated real quadratic form. There are three possibilities:

hyperbolic type: $\mathrm{NS}(X)$ is non-degenerate, of signature $(1,0, \rho(X)-1)$ and $\mathrm{T}(X)$ has signature $\left(2,0, b_{2}(X)-\rho(X)-2\right)$,

parabolic type: $\mathrm{NS}(X) \cap \mathrm{T}(X)$ is of dimension $1, \mathrm{NS}(X)$ has signature $(0,1, \rho(X)-1)$ and $\mathrm{T}(X)$ has signature $\left(2,1, b_{2}(X)-\rho(X)-3\right)$,

elliptic type: $\mathrm{NS}(X)$ is negative definite, of signature $(0,0, \rho(X))$ and $\mathrm{T}(X)$ has signature $\left(3,0, b_{2}(X)-\rho(X)-3\right)$.

By Huybrechts [20, Theorem 3.11], $X$ is projective if and only if $\operatorname{NS}(X)$ is hyperbolic.

Let $G \subset \operatorname{Aut}(X)$ be a finite group of automorphisms of prime order $p$ and fix a generator $g \in G$. If $g^{*} \omega_{X}=\omega_{X}$ then $G$ is called symplectic. Otherwise, there exists a primitive $p$-th root of the unity $\xi_{p}$ such that $g^{*} \omega_{X}=\xi_{p} \omega_{X}$ and $G$ is called non-symplectic. For simplicity we put:

$$
\mathrm{T}_{G}(X):=\mathrm{T}_{G}^{2}(X), \quad \mathrm{S}_{G}(X):=\mathrm{S}_{G}^{2}(X), \quad \mathrm{a}_{G}(X):=\mathrm{a}_{G}^{2}(X), \quad \mathrm{m}_{G}(X):=\mathrm{m}_{G}^{2}(X) .
$$

Lemma 6.1. If $p \leq 19$ then $\mathrm{S}_{G}(X)=\mathrm{T}_{G}(X)^{\perp}$ and $\mathrm{T}_{G}(X)$ is non-degenerate.

Proof. We already know that $\mathrm{T}_{G}(X) \cap \mathrm{S}_{G}(X)=\{0\}$ since $H^{2}(X, \mathbb{Z})$ is torsionfree. Take $x \in \mathrm{T}_{G}(X)$ and $y \in \mathrm{S}_{G}(X)$. Using the $G$-invariance of the bilinear form one gets $\langle x, y\rangle=\left\langle x, g^{i} y\right\rangle$ for all $i$, hence $p\langle x, y\rangle=\langle x, \sigma(y)\rangle=0$ so $\mathrm{S}_{G}(X) \subset$ $\mathrm{T}_{G}(X)^{\perp}$. By Lemma 5.3 one has $\operatorname{rank} \mathrm{T}_{G}(X)+\operatorname{rank} \mathrm{S}_{G}(X)=b_{2}(X)$. Since the lattice $H^{2}(X, \mathbb{Z})$ is non-degenerate, we also have $\operatorname{rank} \mathrm{T}_{G}(X)+\operatorname{rank} \mathrm{T}_{G}(X)^{\perp}=$ $b_{2}(X)$. It follows that $\mathrm{S}_{G}(X)=\mathrm{T}_{G}(X)^{\perp}$ and that the lattice $\mathrm{T}_{G}(X)$ is nondegenerate.

Assume that $G$ is non-symplectic. Then $X$ is algebraic [3]. If 1 is an eigenvalue of $g$ on $\mathrm{T}(X) \otimes \mathbb{C}$ and $t \in \mathrm{T}(X)$ is an eigenvector, one computes:

$$
\left\langle\omega_{X}, t\right\rangle=\left\langle g^{*} \omega_{X}, g^{*} t\right\rangle=\xi_{p}\left\langle\omega_{X}, t\right\rangle
$$

so $t \in \mathrm{T}(X) \cap \mathrm{NS}(X)=\{0\}$, contradiction. One deduces that the eigenvalues of the action of $g$ on $\mathrm{T}(X)$ are primitive $p$-th roots of the unity, so $\mathrm{T}(X) \subset \mathrm{S}_{G}(X)$. 
The minimal polynomial of $g$ on $\mathrm{T}(X)$ is the cyclotomic polynomial $\Phi_{p}$ hence $\varphi(p) \leq b_{2}(X)-\rho(X)$.

Assume now that $G$ is symplectic and $X$ is algebraic or of elliptic type. If $t \in \mathrm{T}(X)$, one gets:

$$
\left\langle\omega_{X}, t\right\rangle=\left\langle\omega_{X}, g^{*} t\right\rangle
$$

so $g^{*} t-t \in \omega_{X}^{\perp}$. Since $X$ is algebraic, $\mathrm{T}(X) \cap \mathrm{NS}(X)=\{0\}$ so $g^{*} t=t$. Then $g$ acts trivially on $\mathrm{T}(X)$, hence $\mathrm{S}_{G}(X) \subset \mathrm{NS}(X)$ and $\varphi(p) \leq \rho(X)$. This property remains true for the parabolic type if $X$ is isomorphic to the Hilbert scheme $S^{[n]}$ of $n$ points on a K3 surface $S$ with the following argument. There exists an injective morphism $\iota: H^{2}(S, \mathbb{C}) \rightarrow H^{2}\left(S^{[n]}, \mathbb{C}\right)$ such that $H^{2}\left(S^{[n]}, \mathbb{Z}\right)=\iota\left(H^{2}(S, \mathbb{Z})\right) \oplus \mathbb{Z} \delta$, where $2 \delta$ is the class of the exceptional divisor of $S^{[n]}$. After normalisation, the form $q$ satisfies $q(\iota(\alpha))=\alpha^{2}$ for $\alpha \in H^{2}(S, \mathbb{Z}), q(\delta)=-2(n-1)$ and $\delta$ is orthogonal to $\iota\left(H^{2}(S, \mathbb{Z})\right)$ (see [3, Proposition 6]). Observe that $\mathrm{NS}\left(S^{[n]}\right)=\iota(\mathrm{NS}(S)) \oplus \mathbb{Z} \delta$ and $\mathrm{T}\left(S^{[n]}\right)=\iota(\mathrm{T}(S))$. Setting $F:=\mathrm{T}\left(S^{[n]}\right) \cap \mathrm{NS}\left(S^{[n]}\right) \cong \mathbb{Z}$, the previous argument shows that $G$ acts trivially on $\mathrm{T}\left(S^{[n]}\right) / F$. Let $c$ be a generator of $F$. Then $c$ is of the form $c=\iota\left(c_{0}\right)$ with $c_{0} \in \mathrm{T}(S) \cap \mathrm{NS}(S)$ and $q(c)=c_{0}^{2}=0$ hence by the Riemann-Roch theorem, $c_{0}$ or $-c_{0}$ is an effective divisor. Changing $c$ to $-c$ if necessary, one can assume that $c_{0}$ is effective. Then $c$ is also an effective divisor hence $g^{*} c=c$. So $g^{*}$ acts trivially on $F$ and $\mathrm{T}\left(S^{[n]}\right) / F$ hence all its eigenvalues on $\mathrm{T}\left(S^{[n]}\right)$ are equal to one. Since $g$ is of finite order, $g^{*}$ is diagonalisable hence finally $g^{*}$ acts as the identity on $\mathrm{T}\left(S^{[n]}\right)$.

6.3. K3 surfaces. In this section, we assume that $X$ is a K3 surface. If $G$ is nonsymplectic, then $\rho(X) \leq 21$ so $p \leq 19$. Nikulin 35 proved that for a symplectic action, one has in fact $p \leq 7$. The lattice $H^{2}(X, \mathbb{Z})$ is unimodular, hence the lattices $\mathrm{T}_{G}(X)$ and $\mathrm{S}_{G}(X)$ are $p$-elementary. Indeed, the generator $g$ of $G$ acts trivially on the discriminant groups $A_{\mathrm{T}_{G}(X)} \cong A_{\mathrm{S}_{G}(X)}$ but $\sigma$ is zero on $A_{\mathrm{S}_{G}(X)}$ hence for all $x \in A_{\mathrm{S}_{G}(X)}$ one has $p x=0$, so these discriminant groups are $p$-torsion groups. It follows that the integer $\mathrm{a}_{G}(X)$ has an important characterization:

$$
\begin{aligned}
& \operatorname{disc}\left(\mathrm{T}_{G}(X)\right)=\operatorname{disc}\left(\mathrm{S}_{G}(X)\right)=p^{\mathrm{a}_{G}(X)}, \\
& \text { so } \mathrm{a}_{G}(X)=a\left(\mathrm{~T}_{G}(X)\right) .
\end{aligned}
$$

Corollary 6.2. Let $X$ be a $K 3$ surface and $G$ a group of automorphisms of prime order $p$. Then one has:

$$
h^{*}\left(X^{G}, \mathbb{F}_{p}\right)= \begin{cases}24-2 \mathrm{a}_{G}(X) & \text { if } p=2 \text { and } X^{G} \neq \emptyset, \\ 24-(p-2) \mathrm{m}_{G}(X)-2 \mathrm{a}_{G}(X) & \text { if } p \geq 3 .\end{cases}
$$

Proof. For $p=2$, if the group $G$ acts symplectically on $X$, by the holomorphic Lefschetz fixed point formula there are 8 isolated fixed points. By Proposition 4.5. the spectral sequence of equivariant cohomology with coefficients in $\mathbb{F}_{p}$ degenerates, so the formula is a consequence of Corollary 4.3. Proposition 5.1 and Corollary 5.8. If $G$ acts non-symplectically and has fixed points, the same argument as above applies. Alternatively, one can observe that since $G$ acts locally at fixed points by quasi-reflections, by a result of Chevalley the quotient $X / G$ is smooth, so $H^{\text {odd }}\left(X / G, \mathbb{F}_{p}\right)=0$ and Proposition 7.6 (see below) gives the result.

For $p \geq 3$, by the holomorphic Lefschetz fixed point formula the fixed locus is never empty so as above the spectral sequence degenerates and the result follows. 
Remark 6.3. This formula does not apply for $p=2$ when $X^{G}=\emptyset$ : in this case, the quotient $X / G$ is an Enriques surface and it is well-known that $\mathrm{a}_{G}(X)=10$.

Remark 6.4. This formula due to Kharlamov [21 for $p=2$ and Artebani-SartiTaki [2] for $p \geq 3$. In both papers, it is obtained by using the classical theory of Smith sequences, under the assumption that $G$ acts non-symplectically. However, there is a small gap in the argument of Artebani-Sarti-Taki [2]: if the fixed locus contains no isolated fixed points, then as above the quotient $X / G$ is smooth so $H^{\text {odd }}\left(X / G, \mathbb{F}_{p}\right)=0$ and as in 2] Proposition 7.8 below gives an equality. Otherwise the cohomology of the quotient may have $p$-torsion: for example, if the fixed locus contains only isolated fixed points, the long cohomology exact sequence of the pair $\left(X / G, X^{G}\right)$ gives $H^{3}\left(X / G, \mathbb{F}_{p}\right) \cong \mathbb{F}_{p}$ so Proposition 7.8 gives only an inequality. Our argument using the degeneracy of the spectral sequence solves this problem.

6.4. Hilbert scheme of two points. Let $S$ be a K3 surface and assume that $X$ is deformation equivalent to the Hilbert scheme $S^{[2]}$ of two points on $S$. Then $1 \leq \rho(X) \leq 21$ so $p \leq 23$. Note that since $\rho\left(S^{[n]}\right) \geq 2$, one has $p \leq 19$ if $X=S^{[n]}$. If $X$ is deformation equivalent to $S^{[n]}$ and $\rho(X)=1$ then the existence of non-symplectic automorphisms of order $p=23$ is not excluded. For a symplectic action on a deformation of $S^{[2]}$, Mongardi 31 shows that $p \leq 11$ so higher order automorphisms on deformations of $S^{[2]}$ are always non-symplectic.

The following lemma is a direct generalisation of Nikulin's results [35, very close from those stated in Mongardi [32].

Lemma 6.5. Assume that $X$ is deformation equivalent to $S^{[2]}$ and that $G$ is an order $p$ group of automorphisms of $X$ with $3 \leq p \leq 19$. Then the lattice $\mathrm{S}_{G}(X)$ has discriminant $A_{\mathrm{S}_{G}(X)} \cong\left(\frac{\mathbb{Z}}{p \mathbb{Z}}\right)^{\mathrm{a}_{G}(X)}$ and in the symplectic case contains no $(-2)$-classes. The invariant lattice $\mathrm{T}_{G}(X)$ has discriminant $A_{\mathrm{T}_{G}(X)} \cong\left(\frac{\mathbb{Z}}{2 \mathbb{Z}}\right) \oplus$ $\left(\frac{\mathbb{Z}}{p \mathbb{Z}}\right)^{\mathrm{a}_{G}(X)}$. Moreover, if $G$ acts symplectically then $\mathrm{S}_{G}(X)$ is negative definite of rank $(p-1) \mathrm{m}_{G}(X)$ and $\mathrm{T}_{G}(X)$ has signature $\left(3,20-(p-1) \mathrm{m}_{G}(X)\right)$. If $G$ acts non-symplectically, then $\mathrm{S}_{G}(X)$ has signature $\left(2,(p-1) \mathrm{m}_{G}(X)-2\right)$ and $\mathrm{T}_{G}(X)$ has signature $\left(1,22-(p-1) \mathrm{m}_{G}(X)\right)$.

Proof. For the fact $\mathrm{S}_{G}(X)$ contains no $(-2)$ classes when the action is symplectic, see Mongardi [32. By Lemma 5.3, from the relation

$$
\left[H^{2}(X, \mathbb{Z}): \mathrm{T}_{G}(X) \oplus \mathrm{S}_{G}(X)\right]^{2}=\operatorname{disc}\left(\mathrm{T}_{G}(X)\right) \cdot \operatorname{disc}\left(\mathrm{S}_{G}(X)\right) \cdot \operatorname{disc}\left(H^{2}(X, \mathbb{Z})\right)^{-1}
$$

we get the formula $\operatorname{disc}\left(\mathrm{T}_{G}(X)\right) \cdot \operatorname{disc}\left(\mathrm{S}_{G}(X)\right)=2 p^{2 \mathrm{a}_{G}(X)}$, hence $\operatorname{disc}\left(\mathrm{S}_{G}(X)\right)=$ $2^{\epsilon} p^{\alpha}$ and $\operatorname{disc}\left(\mathrm{T}_{G}(X)\right)=2^{1-\epsilon} p^{\beta}$ with $\epsilon \in\{0,1\}$ since $p$ is odd, with $\alpha+\beta=2 \mathrm{a}_{G}(X)$. Since the inclusion $\mathrm{T}_{G}(X) \subset H^{2}(X, \mathbb{Z})$ is primitive, as explained in Nikulin [36, §5] the inclusion

$$
M:=\frac{H^{2}(X, \mathbb{Z})}{\mathrm{T}_{G}(X) \oplus \mathrm{S}_{G}(X)} \subset A_{\mathrm{T}_{G}(X)} \oplus A_{\mathrm{S}_{G}(X)}
$$

is such that the projections $p: M \rightarrow A_{\mathrm{T}_{G}(X)}$ and $q: M \rightarrow A_{\mathrm{S}_{G}(X)}$ are $G$-equivariant monomorphisms. We deduce that $\mathrm{a}_{G}(X) \leq \alpha$ and $\mathrm{a}_{G}(X) \leq \beta$. This shows that $\alpha=\beta=\mathrm{a}_{G}(X)$.

We show now that $G$ acts trivially on $A_{\mathrm{S}_{G}(X)}$. There are two possibilities:

(1) $M \cong A_{\mathrm{T}_{G}(X)}$ and $A_{\mathrm{S}_{G}(X)} / M \cong \mathbb{Z} / 2 \mathbb{Z}$,

(2) $M \cong A_{\mathrm{S}_{G}(X)}$ and $A_{\mathrm{T}_{G}(X)} / M \cong \mathbb{Z} / 2 \mathbb{Z}$. 
By Remark 5.4, $M \cong\left(\frac{\mathbb{Z}}{p \mathbb{Z}}\right)^{\mathrm{a}_{G}(X)}$ is a trivial $G$-module so in case (2) the result is clear. In case (11) one has a $G$-equivariant inclusion

$$
M=\left(\frac{\mathbb{Z}}{p \mathbb{Z}}\right)^{\mathrm{a}_{G}(X)} \rightarrow\left(\frac{\mathbb{Z}}{2 \mathbb{Z}}\right) \oplus\left(\frac{\mathbb{Z}}{p \mathbb{Z}}\right)^{\mathrm{a}_{G}(X)}=A_{\mathrm{S}_{G}(X)}
$$

Since $p$ is odd, this map is trivial on the first factor. Since $M$ is a trivial $G$-module this shows that $G$ acts trivially on $A_{\mathrm{S}_{G}(X)}$.

Since $\mathrm{S}_{G}(X)=\operatorname{Ker}(\sigma)$ and $G$ acts trivially on $A_{\mathrm{S}_{G}(X)}$ it follows that $\mathrm{S}_{G}(X)$ is $p$-elementary so $\epsilon=0$. This shows that case (11) cannot occur so we have $M \cong$ $A_{\mathrm{S}_{G}(X)} \cong\left(\frac{\mathbb{Z}}{p \mathbb{Z}}\right)^{\mathrm{a}_{G}(X)}$ and $A_{\mathrm{T}_{G}(X)} \cong\left(\frac{\mathbb{Z}}{2 \mathbb{Z}}\right) \oplus\left(\frac{\mathbb{Z}}{p \mathbb{Z}}\right)^{\mathrm{a}_{G}(X)}$.

If $G$ acts symplectically, the invariant lattice $\mathrm{T}_{G}(X) \otimes_{\mathbb{Z}} \mathbb{C}$ contains the symplectic form $\omega_{X}$, its conjugate $\overline{\omega_{X}}$ and an invariant Kähler class. Since $H^{2}(X, \mathbb{Z})$ has signature $(3,20)$, this implies that $\mathrm{T}_{G}(X)$ has signature $\left(3,20-(p-1) \mathrm{m}_{G}(X)\right)$ and $\mathrm{S}_{G}(X)$ has signature $\left(0,(p-1) \mathrm{m}_{G}(X)\right)$. If $G$ acts non symplectically, then $\omega_{X}, \overline{\omega_{X}} \in$ $\mathrm{S}_{G}(X) \otimes \mathbb{C}$ and $\mathrm{T}_{G}(X)$ still contains an invariant Kähler class, hence $\mathrm{S}_{G}(X)$ has signature $\left(2,(p-1) \mathrm{m}_{G}(X)-2\right)$ and $\mathrm{T}_{G}(X)$ has signature $\left(1,22-(p-1) \mathrm{m}_{G}(X)\right)$.

Markman [27] proved that $H^{\text {odd }}(X, \mathbb{Z})=0$ and $H^{\text {even }}(X, \mathbb{Z})$ is torsion-free, and Verbitsky 40] proved that the cup product map $\operatorname{Sym}^{2} H^{2}(X, \mathbb{Q}) \rightarrow H^{4}(X, \mathbb{Q})$ is an isomorphism. We first study the embedding $\operatorname{Sym}^{2} H^{2}(X, \mathbb{Z}) \hookrightarrow H^{4}(X, \mathbb{Z})$.

Proposition 6.6. If $X$ is deformation equivalent to $S^{[2]}$, then:

$$
\frac{H^{4}(X, \mathbb{Z})}{\operatorname{Sym}^{2} H^{2}(X, \mathbb{Z})} \cong\left(\frac{\mathbb{Z}}{2 \mathbb{Z}}\right)^{\oplus 23} \oplus\left(\frac{\mathbb{Z}}{5 \mathbb{Z}}\right) \text {. }
$$

Proof. It is enough to do the computation for $X=S^{[2]}$. Following an observation of O'Grady [37], we define on $\operatorname{Sym}^{2} H^{2}(X, \mathbb{Z})$ a bilinear symmetric pairing by

$$
\ll \alpha_{1} \odot \alpha_{2}, \alpha_{3} \odot \alpha_{4} \gg:=\left\langle\alpha_{1}, \alpha_{2}\right\rangle\left\langle\alpha_{3}, \alpha_{4}\right\rangle+\left\langle\alpha_{1}, \alpha_{3}\right\rangle\left\langle\alpha_{2}, \alpha_{4}\right\rangle+\left\langle\alpha_{1}, \alpha_{4}\right\rangle\left\langle\alpha_{2}, \alpha_{3}\right\rangle
$$

for $\alpha_{1}, \alpha_{2}, \alpha_{3} \alpha_{4} \in H^{2}(X, \mathbb{Z})$. Note that for any $\alpha \in H^{2}(X, \mathbb{Z})$ one has

$$
\ll \alpha \odot \alpha, \alpha \odot \alpha \gg=3\langle\alpha, \alpha\rangle=\int_{X} \alpha^{4} .
$$

It follows that $\left(\operatorname{Sym}^{2} H^{2}(X, \mathbb{Z}), \ll \cdot, \cdot \gg\right)$ is a sublattice of $H^{4}(X, \mathbb{Z})$ equipped with the Poincaré pairing. Since this lattice is unimodular, one has

$$
\left[H^{4}(X, \mathbb{Z}), \operatorname{Sym}^{2} H^{2}(X, \mathbb{Z})\right]^{2}=\operatorname{disc}\left(\operatorname{Sym}^{2} H^{2}(X, \mathbb{Z})\right) .
$$

Using that $H^{2}(X, \mathbb{Z})$ is isometric to $U^{\oplus 3} \oplus E_{8}(-1)^{\oplus 2} \oplus\langle-2\rangle$ it is easy to compute that the $\operatorname{Sym}^{2} H^{2}(X, \mathbb{Z})$ has discriminant $\operatorname{disc}\left(\operatorname{Sym}^{2} H^{2}(X, \mathbb{Z})\right)=2^{46} \cdot 5^{2}$. The result follows.

Remark 6.7. In order to understand the (somehow surprising) 5-torsion class, one can perform an explicit computation as follows. For $\alpha \in H^{*}(S, \mathbb{Z})$ and $i \in \mathbb{Z}$, we denote by $\mathfrak{q}_{i}(\alpha) \in \operatorname{End}\left(H^{*}(X, \mathbb{Z})\right)$ the Nakajima operators $[33$ and by $|0\rangle \in$ $H^{0}\left(S^{[0]}, \mathbb{Z}\right)$ the unit. Let $\left(\alpha_{i}\right)_{i=1, \ldots, 22}$ be an integral basis of $H^{2}(S, \mathbb{Z})$, denote by $1 \in H^{0}(S, \mathbb{Z})$ the unit and by $x \in H^{4}(S, \mathbb{Z})$ the class of a point. The results of Qin-Wang [39, Theorem 5.4, Remark 5.6] give the following integral basis:

- integral basis of $H^{2}(X, \mathbb{Z}): \frac{1}{2} \mathfrak{q}_{2}(1)|0\rangle, \mathfrak{q}_{1}(1) \mathfrak{q}_{1}\left(\alpha_{i}\right)|0\rangle$, 
- integral basis of $H^{4}(X, \mathbb{Z})$ :

$$
\begin{array}{rr}
\mathfrak{q}_{1}(1) \mathfrak{q}_{1}(x)|0\rangle, & \mathfrak{q}_{2}\left(\alpha_{i}\right)|0\rangle, \quad \mathfrak{q}_{1}\left(\alpha_{i}\right) \mathfrak{q}_{1}\left(\alpha_{j}\right)|0\rangle \text { with } i<j, \\
& \mathfrak{m}_{1,1}\left(\alpha_{i}\right)|0\rangle=\frac{1}{2}\left(\mathfrak{q}_{1}\left(\alpha_{i}\right)^{2}-\mathfrak{q}_{2}\left(\alpha_{i}\right)\right)|0\rangle .
\end{array}
$$

The cup product map $\operatorname{Sym}^{2} H^{2}(X, \mathbb{Q}) \rightarrow H^{4}(X, \mathbb{Q})$ can be computed explicitly by using the algebraic model constructed by Lehn-Sorger [24]:

- for $\alpha \in H^{2}(S, \mathbb{Z}): \frac{1}{2} \mathfrak{q}_{2}(1)|0\rangle \cup \mathfrak{q}_{1}(1) \mathfrak{q}_{1}(\alpha)|0\rangle=\mathfrak{q}_{2}(\alpha)|0\rangle$,

- for $\alpha, \beta \in H^{2}(S, \mathbb{Z})$ :

$$
\mathfrak{q}_{1}(1) \mathfrak{q}_{1}(\alpha)|0\rangle \cup \mathfrak{q}_{1}(1) \mathfrak{q}_{1}(\beta)|0\rangle=\left(\int_{S} \alpha \beta\right) \mathfrak{q}_{1}(1) \mathfrak{q}_{1}(x)|0\rangle+\mathfrak{q}_{1}(\alpha) \mathfrak{q}_{1}(\beta)|0\rangle,
$$

- denote by $\delta: S \rightarrow S \times S$ the diagonal embedding. We denote the push-forward map followed by the Künneth isomorphism by $\delta_{*}: H^{*}(S, \mathbb{Z}) \rightarrow H^{*}(S, \mathbb{Z}) \otimes H^{*}(S, \mathbb{Z})$. Writing $\delta_{*} 1=\sum_{i, j} \mu_{i, j} \alpha_{i} \otimes \alpha_{j}+1 \otimes x+x \otimes 1$ for some $\mu_{i, j} \in \mathbb{Z}$ with the property that $\mu_{i, j}=\mu_{j, i}$, one has:

$$
\frac{1}{2} \mathfrak{q}_{2}(1)|0\rangle \cup \frac{1}{2} \mathfrak{q}_{2}(1)|0\rangle=\sum_{i<j} \mu_{i, j} \mathfrak{q}_{1}\left(\alpha_{i}\right) \mathfrak{q}_{1}\left(\alpha_{j}\right)|0\rangle+\frac{1}{2} \sum_{i} \mu_{i, i} \mathfrak{q}_{1}\left(\alpha_{i}\right)^{2}|0\rangle+\mathfrak{q}_{1}(1) \mathfrak{q}_{1}(x)|0\rangle
$$

An elementary computation (with the help of a computer) allows to determine the 253 coefficients $\mu_{i, j}$, by using the fact that $\delta_{*}$ is the adjoint of the cup-product and the intersection matrix of $H^{2}(S, \mathbb{Z}) \cong U^{\oplus 3} \oplus E_{8}(-1)^{\oplus 2}$ (where $U$ denotes the rank 2 hyperbolic lattice). One can then express the basis of $\operatorname{Sym}^{2} H^{2}(X, \mathbb{Z})$ in terms of the given integral basis of $H^{4}(X, \mathbb{Z})$ and compute the Smith normal form of the quotient $\frac{H^{4}(X, \mathbb{Z})}{\operatorname{Sym}^{2} H^{2}(X, \mathbb{Z})}$. One finds $\left(\frac{\mathbb{Z}}{2 \mathbb{Z}}\right)^{\oplus 22} \oplus\left(\frac{\mathbb{Z}}{10 \mathbb{Z}}\right)$.

A precise look at the computation of the Smith normal form shows that the 2-divisible classes in $\operatorname{Sym}^{2} H^{2}(X, \mathbb{Z})$ are the six vectors $\mathfrak{m}_{1,1}\left(\alpha_{i}\right)|0\rangle$ for the basis elements $\alpha_{i} \in U^{\oplus 3}$ and the 16 vectors $\mathfrak{m}_{1,1}\left(\alpha_{i}\right)|0\rangle-\mathfrak{q}_{1}(1) \mathfrak{q}_{1}(x)|0\rangle$ for the basis elements $\alpha_{i} \in E_{8}(-1)^{\oplus 2}$. Finally the 10-divisible class is $\mathfrak{q}_{1}(1) \mathfrak{q}_{1}(x)|0\rangle$.

Remark 6.8. If $X$ is deformation equivalent to $S^{[n]}$ with $n \geq 4$, Markman [28, Theorem 1.10] (see also [26, Theorem 9.3]) proved that the quotient $\frac{H^{4}\left(S^{[n]}, \mathbb{Z}\right)}{\operatorname{Sym}^{2} H^{2}\left(S^{[n]}, \mathbb{Z}\right)}$ is free of rank 24 . The case $n=3$ remains to be computed.

Let $S$ be a K3 surface and $G$ a finite group of automorphisms of prime order $p$ on $S^{[2]}$. We give some degeneracy results of the spectral sequence of equivariant cohomology with coefficients in $\mathbb{F}_{p}$.

Lemma 6.9. Denote by $e:=2 \delta$ the class of the exceptional divisor of $S^{[2]}$. Then $\int_{S^{[2]}} e^{4}=2^{6} \cdot 3$ and the map $H^{2}\left(S^{[2]}, \mathbb{Z}\right) \rightarrow H^{6}\left(S^{[2]}, \mathbb{Z}\right), \beta \mapsto e^{2} \beta$ has discriminant $2^{70} \cdot 3$.

Proof. By Beauville [4] and Fujiki [17 there exists a constant $c_{S^{[2]}}$ such that

$$
\int_{S^{[2]}} e^{4}=c_{S^{[2]}} q(e)^{2} .
$$

One has $q(e)=-8$ and by Markushevich [29, Proposition 1.2] we have $c_{S^{[2]}}=3$, so $\int_{S^{[2]}} e^{4}=2^{6} \cdot 3$. The multiplication map $H^{2}\left(S^{[2]}, \mathbb{Z}\right) \rightarrow H^{6}\left(S^{[2]}, \mathbb{Z}\right), \beta \mapsto e^{2} \beta$ is 
equivalent by Poincaré duality to the bilinear form $H^{2}\left(S^{[2]}, \mathbb{Z}\right) \times H^{2}\left(S^{[2]}, \mathbb{Z}\right) \rightarrow \mathbb{Z}$, $(\alpha, \beta) \mapsto \int_{S^{[2]}} \alpha e^{2} \beta$. From the formal relation in $\mathbb{Z}[x, y, z]$

$$
\int_{S^{[2]}}(\alpha x+e y+\beta z)^{4}=3 q(\alpha x+e y+\beta z)^{2},
$$

and by extracting the coefficient of $x y^{2} z$ we get the formula

$$
\int_{S^{[2]}} \alpha e^{2} \beta=-8\langle\alpha, \beta\rangle+2\langle\alpha, e\rangle\langle e, \beta\rangle .
$$

Denoting by $\Lambda:=E_{8}(-1)^{\oplus 2} \oplus U^{\oplus 3}$ the $\mathrm{K} 3$ lattice (the lattice $H^{2}(S, \mathbb{Z})$ for the intersection product), the lattice $H^{2}\left(S^{[2]}, \mathbb{Z}\right) \cong H^{2}(S, \mathbb{Z}) \oplus \mathbb{Z} \delta$ equiped with this bilinear form is then isometric to $\Lambda(-8) \oplus\langle 48\rangle$, so its discriminant is $2^{70} \cdot 3$.

Lemma 6.10. Denote by $c_{2}\left(S^{[2]}\right)$ the second Chern class of the tangent bundle on $S^{[2]}$. Then $\int_{S^{[2]}} c_{2}\left(S^{[2]}\right)^{2}=2^{2} \cdot 3^{2} \cdot 23$ and the multiplication map

$$
H^{2}\left(S^{[2]}, \mathbb{Z}\right) \rightarrow H^{6}\left(S^{[2]}, \mathbb{Z}\right), \beta \mapsto c_{2}\left(S^{[2]}\right) \beta
$$

has discriminant ${ }^{24} \cdot 3^{23} \cdot 5^{23}$.

Proof. The Chern number $\int_{S^{[2]}} c_{2}\left(S^{[2]}\right)^{2}=828=2^{2} \cdot 3^{2} \cdot 23$ is well-known (see Ellingsrud-Göttsche-Lehn [16] or Nieper-Wisskirchen [34, Remark 4.13]). To compute the discriminant of the multiplication map, similarly as in the proof of the previous lemma we consider the quadratic form $H^{2}\left(S^{[2]}, \mathbb{Z}\right) \rightarrow \mathbb{Z}, \alpha \mapsto c_{2}\left(S^{[2]}\right) \alpha^{2}$. By Nieper-Wisskirchen [34, Corollary 3.8] one has

$$
\begin{aligned}
\int_{S^{[2]}} c_{2}\left(S^{[2]}\right) \alpha^{2} & =96 \lambda(\alpha) \operatorname{td}^{\frac{1}{2}}\left(S^{[2]}\right), \\
\int_{S^{[2]}} \alpha^{4} & =24 \lambda(\alpha)^{2} \operatorname{td}^{\frac{1}{2}}\left(S^{[2]}\right) .
\end{aligned}
$$

Recall that $\int_{S^{[2]}} \alpha^{4}=3 q(\alpha)^{2}$ and that the square root of the Todd genus is here:

$$
\sqrt{\operatorname{td}}\left(S^{[2]}\right)=1+\frac{1}{24} c_{2}\left(S^{[2]}\right)+\frac{7}{5760} c_{2}\left(S^{[2]}\right)^{2}-\frac{1}{1440} c_{4}\left(S^{[2]}\right) .
$$

Using that $c_{4}\left(S^{[2]}\right)=324$ (see [34]) one gets $\operatorname{td}^{\frac{1}{2}}\left(S^{[2]}\right):=\int_{S^{[2]}} \sqrt{\operatorname{td}}\left(S^{[2]}\right)=\frac{25}{32}$. Putting all together we get

$$
\left(\int_{S^{[2]}} c_{2}\left(S^{[2]}\right) \alpha^{2}\right)^{2}=900 q(\alpha)^{2}
$$

so $\int_{S^{[2]}} c_{2}\left(S^{[2]}\right) \alpha^{2}= \pm 30 q(\alpha)$. For this quadratic form, the lattice $H^{2}\left(S^{[2]}, \mathbb{Z}\right)$ is then isometric to $\Lambda(30) \oplus\langle-60\rangle$, so the multiplication map has discriminant $2^{24} \cdot 3^{23} \cdot 5^{23}$.

Let $\left(e_{i}\right)_{i}$ be an orthonormal basis of $H^{2}(X, \mathbb{C})$ for $q$. The image of the class $\sum_{i} e_{i} \cdot e_{i} \in \operatorname{Sym}^{2} H^{2}(X, \mathbb{C})$ in $H^{4}(X, \mathbb{C})$ is denoted $q^{-1}$ and called the BeauvilleBogomolov class. By Markman [25, for $X$ deformation equivalent to $S^{[n]}$ there is a decomposition

$$
q^{-1}=c_{2}(X)+2 \kappa_{2}\left(E_{x}\right)
$$

where $x \in X$ is a point, $E_{x}$ a rank $2 n-2$ reflexive coherent twisted sheaf and $\kappa\left(E_{x}\right):=\operatorname{ch}\left(E_{x}\right) \exp \left(\frac{-c_{1}\left(E_{x}\right)}{2 n-2}\right)$. Since $\kappa_{2}=\frac{c_{1}^{2}}{8}-c_{2}$, the class $u:=4 q^{-1}$ lives in $H^{4}\left(S^{[2]}, \mathbb{Z}\right)$. As noted by O'Grady [37] and Markman [25], for $n=2$ the classes 
$q^{-1}, c_{2}(X)$ and $\kappa_{2}\left(E_{x}\right)$ span a 1 -dimensional space and in fact $q^{-1}=\frac{5}{6} c_{2}(X)$ in $H^{2}(X, \mathbb{Q})$.

Lemma 6.11. Assume that $X$ is deformation equivalent to $S^{[2]}$. One has $\int_{X} u^{2}=$ $2^{4} \cdot 5^{2} \cdot 23$ and the map $H^{2}(X, \mathbb{Z}) \rightarrow H^{6}(X, \mathbb{Z}), \beta \mapsto u \beta$ has discriminant $2^{47} \cdot 5^{46}$.

Proof. This is a direct consequence of the equality $3 u=10 c_{2}(X)$ in $H^{2}(X, \mathbb{Z})$. Here is an alternative, more direct argument. Let $\left(e_{i}\right)_{i}$ be an orthonormal basis of $H^{2}(X, \mathbb{C})$ for $q, v:=\sum_{i} x_{i} e_{i} \in H^{2}(X, \mathbb{C})$ and $\alpha \in H^{2}(X, \mathbb{C})$. From the relation

$$
\int_{X}(v+\alpha)^{4}=3 q(v+\alpha)^{2}
$$

and by extracting the quadratic part in $\alpha$ we get

$$
\int_{X} v^{2} \alpha^{2}=q(v) q(\alpha)+2\langle v, \alpha\rangle^{2} .
$$

By extracting the square coefficients in the variables $x_{i}$ and putting them to one we get

$$
\begin{aligned}
\int_{X}\left(\sum_{i} e_{i}^{2}\right) \alpha^{2} & =q\left(\sum_{i} e_{i}\right) q(\alpha)+2 \sum_{i}\left\langle e_{i}, \alpha\right\rangle^{2} \\
\int_{X} q^{-1} \alpha^{2} & =b_{2}(X) q(\alpha)+2 q(\alpha) \\
& =25 q(\alpha)
\end{aligned}
$$

Since $u=4 q^{-1}$ this implies that $\int_{X} u \alpha^{2}=2^{2} 5^{2} q(\alpha)$. For this quadratic form, the lattice $H^{2}(X, \mathbb{Z})$ is then isometric to $\Lambda\left(2^{2} 5^{2}\right) \oplus\left\langle-2^{3} 5^{2}\right\rangle$ so the multiplication map has discriminant $2^{47} \cdot 5^{46}$. Taking $\alpha=\sum_{i} x_{i} e_{i}$, we also have

$$
\int_{X} u\left(\sum_{i} x_{i} e_{i}\right)^{2}=2^{2} 5^{2} q\left(\sum_{i} x_{i} e_{i}\right) \text {. }
$$

By extracting the square coefficients in $x_{i}$ we get similarly

$$
\int_{X} u \sum_{i} e_{i}^{2}=2^{2} 5^{2} \sum_{i} q\left(e_{i}\right)
$$

hence $\int_{X} u^{2}=2^{4} \cdot 5^{2} \cdot 23$.

Proposition 6.12. Let $G$ be an order $p$ group acting on $X$. The spectral sequence of equivariant cohomology with coefficients in $\mathbb{F}_{p}$ degenerates at the $E_{2}$-term in the following cases:

(1) $X$ is deformation equivalent to $S^{[2]}, 3 \leq p \leq 19$ and $p \neq 5$.

(2) $G$ acts by natural automorphisms on $S^{[2]}$ and $p \neq 2$.

Proof.

(11) If $X$ is deformation equivalent to $S^{[2]}$, the class $c_{2}\left(T_{X}\right)$ is $G$-equivariant and can be used in Proposition 4.6. By lemma 6.10 the degeneracy conditions are fullfilled for $p>5$. For $p=3$, by Lemma 6.11 one can use the class $u=4 q^{-1}$ since from the definition of $q^{-1}$ it is clear that this class is $G$-invariant (alternatively, $u$ is proportional to $c_{2}(X)$ in $H^{2}(X, \mathbb{Q})$ so it is $G$-equivariant). 
(21) If $G$ acts by natural automorphisms on $S^{[2]}$, the exceptional divisor is $G$ invariant and it can be used in Proposition 4.6. By Lemma 6.9 the degeneracy conditions are fullfilled if $p>3$. In the case $p=3$ the class $u=4 q^{-1}$ can be used again.

Lemma 6.13. Assume that $G=\mathbb{Z} / 2 \mathbb{Z}$ and let $M$ be a finite-dimensional $\mathbb{F}_{2}[G]$ module. Then:

$$
\begin{aligned}
& \ell_{1}\left(\operatorname{Sym}^{2} M\right)=\frac{\ell_{1}(M)\left(\ell_{1}(M)+1\right)}{2}+\ell_{2}(M), \\
& \ell_{2}\left(\operatorname{Sym}^{2} M\right)=\ell_{2}(M)\left(\ell_{2}(M)+\ell_{1}(M)\right) .
\end{aligned}
$$

Proof. The $\mathbb{F}_{2}[G]$-modules $M$ and $\mathrm{Sym}^{2} M$ decompose as:

$$
\begin{aligned}
M & \cong N_{2}^{\oplus \ell_{2}(M)} \oplus N_{1}^{\oplus \ell_{1}(M)}, \\
\operatorname{Sym}^{2} M & \cong N_{2}^{\oplus \ell_{2}\left(\mathrm{Sym}^{2} M\right)} \oplus N_{1}^{\oplus \ell_{1}\left(\mathrm{Sym}^{2} M\right)} .
\end{aligned}
$$

By elementary matrix computations, one finds the following $G$-module decompositions:

$$
\begin{aligned}
& \operatorname{Sym}^{2} N_{2} \cong N_{2} \oplus N_{1}, \quad N_{2} \otimes N_{2} \cong N_{2}^{\oplus 2}, \quad N_{2} \otimes N_{1} \cong N_{2} \\
& \operatorname{Sym}^{2} N_{1} \cong N_{1}, \quad N_{1} \otimes N_{1} \cong N_{1} \text {. }
\end{aligned}
$$

The result follows.

Lemma 6.14. Assume that $3 \leq p \leq 19, G=\mathbb{Z} / p \mathbb{Z}$ and let $M$ be a finitedimensional $\mathbb{F}_{p}[G]$-module. Then:

$$
\begin{aligned}
\ell_{1}\left(\operatorname{Sym}^{2} M\right)= & \frac{\ell_{1}(M) \cdot\left(\ell_{1}(M)+1\right)}{2}+\frac{\ell_{p-1}(M) \cdot\left(\ell_{p-1}(M)-1\right)}{2} \\
\ell_{p-1}\left(\operatorname{Sym}^{2} M\right)= & \ell_{p-1}(M) \cdot \ell_{1}(M), \\
\ell_{p}\left(\operatorname{Sym}^{2} M\right)= & \frac{p+1}{2} \cdot \ell_{p}(M)+p \cdot \frac{\ell_{p}(M) \cdot\left(\ell_{p}(M)-1\right)}{2}+\frac{p-1}{2} \cdot \ell_{p-1}(M) \\
& +(p-1) \cdot \ell_{p}(M) \cdot \ell_{p-1}(M)+\ell_{p}(M) \cdot \ell_{1}(M) \\
& +(p-2) \cdot \frac{\ell_{p-1}(M) \cdot\left(\ell_{p-1}(M)-1\right)}{2}
\end{aligned}
$$

and $\ell_{i}\left(\operatorname{Sym}^{2} M\right)=0$ for $2 \leq i \leq p-2$.

Proof. As before, we have the decompositions:

$$
\begin{aligned}
M & \cong N_{p}^{\oplus \ell_{p}(M)} \oplus N_{p-1}^{\oplus \ell_{p-1}(M)} \oplus N_{1}^{\oplus \ell_{1}(M)}, \\
\operatorname{Sym}^{2} M & \cong \bigoplus_{1 \leq q \leq p} N_{q}^{\oplus \ell_{q}\left(\operatorname{Sym}^{2} M\right) .}
\end{aligned}
$$

By elementary matrix computations, one finds the following $G$-module decompositions:

$$
\begin{aligned}
& \mathrm{Sym}^{2} N_{p} \cong N_{p}^{\oplus \frac{p+1}{2}}, \quad N_{p} \otimes N_{p} \cong N_{p}^{\oplus p}, \quad N_{p-1} \otimes N_{p-1} \cong N_{p}^{\oplus p-2} \oplus N_{1} \\
& \mathrm{Sym}^{2} N_{p-1} \cong N_{p}^{\oplus \frac{p-1}{2}}, \quad N_{p} \otimes N_{p-1} \cong N_{p}^{\oplus p-1}, \quad N_{p-1} \otimes N_{1} \cong N_{p-1} \\
& \operatorname{Sym}^{2} N_{1} \cong N_{1}, \quad N_{p} \otimes N_{1} \cong N_{p}, \quad N_{1} \otimes N_{1} \cong N_{1} .
\end{aligned}
$$

The result follows. 
Corollary 6.15. Let $X$ be deformation equivalent to $S^{[2]}$ and $G$ be a group of automorphisms of prime order $p$ on $X$ with $3 \leq p \leq 19$ and $p \neq 5$. Then:

$$
\begin{aligned}
h^{*}\left(X^{G}, \mathbb{F}_{p}\right)= & 324-2 \mathrm{a}_{G}(X)\left(25-\mathrm{a}_{G}(X)\right)-(p-2) \mathrm{m}_{G}(X)\left(25-2 \mathrm{a}_{G}(X)\right) \\
& +\frac{1}{2} \mathrm{~m}_{G}(X)\left((p-2)^{2} \mathrm{~m}_{G}(X)-p\right)
\end{aligned}
$$

with

$$
\begin{aligned}
& 2 \leq(p-1) \mathrm{m}_{G}(X)<23, \\
& 0 \leq \mathrm{a}_{G}(X) \leq \min \left\{(p-1) \mathrm{m}_{G}(X), 23-(p-1) \mathrm{m}_{G}(X)\right\} .
\end{aligned}
$$

Proof. By Proposition 6.12 and Corollary 5.12 using Poincaré duality one has:

$$
h^{*}\left(X^{G}, \mathbb{F}_{p}\right)=324-4 \mathrm{a}_{G}(X)-2 \mathrm{a}_{G}^{4}(X)-2(p-2) \mathrm{m}_{G}(X)-(p-2) \mathrm{m}_{G}^{4}(X) .
$$

By Proposition 6.6 one has an isomorphism $\operatorname{Sym}^{2} H^{2}\left(X, \mathbb{F}_{p}\right) \cong H^{4}\left(X, \mathbb{F}_{p}\right)$ so by Lemma 6.14, using Corollaries $5.8 \& 5.10$ one can express the parameters $\mathrm{a}_{G}^{4}(X)$ and $\mathrm{m}_{G}^{4}(X)$ in terms of $\mathrm{a}_{G}(X)$ and $\mathrm{m}_{G}(X)$. One gets easily the formula. The estimates for the parameters $\mathrm{a}_{G}(X), \mathrm{m}_{G}(X)$ come from Proposition 5.13 noting that $p$ is odd and that $\mathrm{m}_{G}(X)$ cannot be zero, otherwise $\mathrm{T}_{G}(X)=H^{2}(X, \mathbb{Z})$ so $G$ acts trivially on $H^{2}(X, \mathbb{Z})$. By Beauville [3, Proposition 10] this is impossible since $G \neq\{$ id $\}$.

Remark 6.16.

(1) If $G$ acts symplectically, the inclusions $\mathrm{T}(X) \subset \mathrm{T}_{G}(X)$ and $\mathrm{S}_{G}(X) \subset$ $\mathrm{NS}(X)$ give one more relation: $(p-1) \mathrm{m}_{G}(X) \leq \rho(X)$. If instead $G$ acts non symplectically, the inclusions $\mathrm{T}(X) \subset \mathrm{S}_{G}(X)$ and $\mathrm{T}_{G}(X) \subset \mathrm{NS}(X)$ give $23-(p-1) \mathrm{m}_{G}(X) \leq \rho(X)$.

(2) Assume that $G$ is an order $p$ group of automorphisms of $S$. We denote also by $G$ the group of natural automorphisms induced on $S^{[2]}$. Since the exceptional divisor of $S^{[2]}$ is invariant by $G$, it is clear that a ${ }_{G}\left(S^{[2]}\right)=\mathrm{a}_{G}(S)$ and $\mathrm{m}_{G}\left(S^{[2]}\right)=\mathrm{m}_{G}(S)$. For example, if $G$ acts symplectically and $p=3$, by Nikulin [35] $G$ has 6 isolated fixed points on $S$ and by Garbagnati-Sarti [18, Theorem 4.1] one has $\mathrm{a}_{G}(S)=6$ and $\mathrm{m}_{G}(S)=6$. By Corollary 6.15 we get $h^{*}\left(S^{[2]}, \mathbb{F}_{5}\right)=27$. In this case it is easy to see that the fixed locus $\left(S^{[2]}\right)^{G}$ consists indeed in 27 isolated points [6, Exemple 2].

(3) If $X$ is deformation equivalent to $S^{[2]}$ and $p \in\{17,19\}$, it follows from the inequalities given in Corollary 6.15 that $\mathrm{m}_{G}(X)=1$. Then necessarily $\mathrm{a}_{G}(X)>0$. Otherwise, by Lemma 6.5 the lattice $\mathrm{S}_{G}(X)$ would be even unimodular of signature $(2,14)$ for $p=19$ or $(2,14)$ for $p=17$. By an theorem of Milnor (see Nikulin [36, Theorem 1.1.1]) such lattices do not exist.

\subsection{Applications.}

\subsubsection{Existence of fixed points.}

Proposition 6.17. Let $X$ be deformation equivalent to $S^{[2]}$ and $G$ be a group of automorphisms of prime order on $X$. Then the fixed locus $X^{G}$ is not empty.

Proof. Denote by $g$ a generator of $G$ and by $\xi_{p}$ a primitive $p$-th root of the unity. If $g$ acts symplectically, its holomorphic Lefschetz number for the sheaf $\mathcal{O}_{X}$ is three so $G$ has fixed points. If $g$ acts non-symplectically, its holomorphic Lefschetz number 
is $1+\xi_{p}+\xi_{p}^{2}$, so $G$ has fixed points if $p \neq 3$. If $p=3$ one can use Corollary 6.15 to check all possible values of $h^{*}\left(X^{G}, \mathbb{F}_{3}\right)$. One finds that it is never zero, so $X^{G}$ is never empty.

Corollary 6.18. Let $X$ be deformation equivalent to $S^{[2]}$ and $G$ be a finite group of automorphisms of $X$. Then $G$ does not act freely on $X$.

Remark 6.19. This result implies that it is not possible to construct Enriques varieties of dimension four and index three, as defined in Boissière-Nieper-WißkirchenSarti [7] and Oguiso-Schroër [38, if one starts with deformations of Hilbert schemes of two points on a K3 surface.

6.5.2. An automorphism of order eleven. Consider the cubic $C$ in $\mathbb{P}^{5}$ given by the equation

$$
x_{0}^{3}+x_{1}^{2} x_{5}+x_{2}^{2} x_{4}+x_{3}^{2} x_{2}+x_{4}^{2} x_{1}+x_{5}^{2} x_{3}=0 .
$$

Let $\xi$ be a primitive eleventh root of the unit and consider the order 11 automorphis $\varphi$ of $C$ given by

$$
\varphi\left(x_{0}, x_{1}, x_{2}, x_{3}, x_{4}, x_{5}\right)=\left(x_{0}, \xi x_{1}, \xi^{3} x_{2}, \xi^{4} x_{3}, \xi^{5} x_{4}, \xi^{9} x_{5}\right) .
$$

As explained in Mongardi [31], $\varphi$ induces a symplectic automorphism of order 11 on the Fano variety of lines $X$ of the cubic fourfold $C$, with 5 isolated fixed points. Using our main formula given in Corollary 6.15, one finds that there is only one possibility for the parameters $\mathrm{a}_{G}(X), \mathrm{m}_{G}(X)$, that is:

$$
\mathrm{a}_{G}(X)=2, \quad \mathrm{~m}_{G}(X)=2,
$$

hence $\rho(X) \in\{20,21\}$. Since $X$ is algebraic, the inclusion $\mathrm{S}_{G}(X) \subset \mathrm{NS}(X)$ is strict since the Néron-Severi lattice is hyperbolic, so $\rho(X)=21$ (see also Mongardi 31] for a more general statement). It follows from Lemma 6.5 that $\mathrm{S}_{G}(X)$ has signature $(0,20)$ and discriminant $A_{\mathrm{S}_{G}(X)} \cong\left(\frac{\mathbb{Z}}{p \mathbb{Z}}\right)^{2}$ and contains no $(-2)$-classes. Furthermore, $\mathrm{T}_{G}(X)$ has signature $(3,0)$ and discriminant $A_{\mathrm{T}_{G}(X)} \cong \frac{\mathbb{Z}}{2 \mathbb{Z}} \oplus\left(\frac{\mathbb{Z}}{p \mathbb{Z}}\right)^{2}$. We can deduce the isometry class of the invariant lattice as follows. From the classification of Brandt-Intrau [9] of positive definite ternary quadratic forms we find that there are two possibilities for the invariant lattice $\mathrm{T}_{G}(X)$, given by the following Gram matrices:

$$
A:=\left(\begin{array}{ccc}
2 & 1 & 0 \\
1 & 6 & 0 \\
0 & 0 & 22
\end{array}\right), \quad B:=\left(\begin{array}{ccc}
6 & 2 & 2 \\
2 & 8 & -3 \\
2 & -3 & 8
\end{array}\right)
$$

(these two lattices have the same discriminant but different discriminant forms).

Denote by $Z \subset X \times C$ the universal family, with projections $p, q$ on $X$ and $C$. By Beauville-Donagi [5], the Abel-Jacobi map

$$
\alpha:=p_{*} q^{*}: H^{4}(C, \mathbb{Z}) \rightarrow H^{2}(X, \mathbb{Z})
$$

is an isomorphism of Hodge structures. Denote by $h \in H^{2}(C, \mathbb{Z})$, resp. $g \in$ $H^{2}(X, \mathbb{Z})$ the hyperplane class of $C$, resp. of $X$ for the Plücker embedding. Denote by $H^{4}(C, \mathbb{Z})_{0}$ the primitive cohomology (the orthogonal of $h$ for the intersection form on $C$ ), and similarly $H^{2}(X, \mathbb{Z})_{0}$ the orthogonal of $g$ for the BeauvilleBogomolov form, denoted $(-,-)_{X}$. By Beauville-Donagi [5], the Abel-Jacobi map induces an isometry between $H^{4}(C, \mathbb{Z})_{0}$ and $H^{2}(X, \mathbb{Z})_{0}$. It follows from Hassett [19] 
that $\operatorname{disc}\left(H^{2}(X, \mathbb{Z})_{0}\right)=3$. Note that $(g, g)_{X}=6$. By Mongardi 31], for the special choice of the cubic $C$ with a symplectic automorphism of order 11 constructed above, one has

$$
\mathrm{NS}(X) \cong(6) \oplus E_{8}(-1)^{2} \oplus\left(\begin{array}{cc}
-2 & 1 \\
1 & -6
\end{array}\right)
$$

hence $\operatorname{disc}(\mathrm{NS}(X))=2 \cdot 3 \cdot 11^{2}$. Since $\operatorname{disc}\left(H^{2}(X, \mathbb{Z})\right)=2$, it follows, with the same argument as above, that $\operatorname{disc}(\mathrm{T}(X)) \in\left\{2^{2} \cdot 3 \cdot 11^{2}, 3 \cdot 11^{2}\right\}$. Now consider $\mathrm{NS}(X)_{0}:=$ $\mathrm{NS}(X) \cap q^{\perp} \subset H^{2}(X, \mathbb{Z})_{0}$ and observe that $\mathrm{T}(X)=\mathrm{T}(X) \cap q^{\perp} \subset H^{2}(X, \mathbb{Z})_{0}$. One has $\operatorname{disc}\left(\mathrm{NS}(X)_{0}\right)=11^{2}$ and $\operatorname{disc}\left(H^{2}(X, \mathbb{Z})_{0}\right)=3$ hence $\operatorname{disc}(\mathrm{T}(X)) \in\left\{11^{2}, 3 \cdot 11^{2}\right\}$. As a consequence, $\operatorname{disc}(\mathrm{T}(X))=3 \cdot 11^{2}$. Now, observe that since $\mathrm{T}_{G}(X)$ has rank 3 and $G$ acts symplectically, one has $\mathrm{T}(X) \subset \mathrm{T}_{G}(X)$ and in fact $\mathrm{T}(X)=\mathrm{T}_{G}(X) \cap q^{\perp}$. From the two Gram matrices $A$ and $B$ it is easy to deduce all elements of square 6 (there are very few) and to compute their orthogonal and its discriminant. One finds that the only possibility giving the right discriminant for $\mathrm{T}(X)$ is the choice of the matrix $B$ (compare with Mongardi 31] for a different argument).

\section{Classical methods in Smith theory}

In this section, we study the case when the spectral sequence does not degenerate. It happens that one gets bounds for $h^{*}\left(X^{G}, \mathbb{F}_{p}\right)$ that are closely related to the previous results, where we see that the defect is contained in the $p$-torsion of the cohomology of the quotient $X / G$.

7.1. Smith exact sequences. Consider the chain complex $C_{*}(X)$ of $X$ with coefficients in $\mathbb{F}_{p}$ and its subcomplexes $\bar{\tau}^{i} C_{*}(X)$ for $1 \leq i \leq p-1$ (with $\bar{\sigma}=\bar{\tau}^{p-1}$ ). The basic tools in Smith theory are the following results, valid for any prime number $p$ :

\section{Proposition 7.1.}

(1) [10, Theorem 3.1] For $1 \leq i \leq p-1$ there is an exact sequence of complexes:

$$
0 \longrightarrow \bar{\tau}^{i} C_{*}(X) \oplus C_{*}\left(X^{G}\right) \stackrel{\iota}{\longrightarrow} C_{*}(X) \stackrel{\bar{\tau}^{p-i}}{\longrightarrow} \bar{\tau}^{p-i} C_{*}(X) \longrightarrow 0
$$

where $\iota$ denotes the sum of the inclusions.

(2) [10, p.125] For $1 \leq i \leq p-1$ there is an exact sequence of complexes:

$$
0 \longrightarrow \bar{\sigma} C_{*}(X) \stackrel{\iota}{\longrightarrow} \bar{\tau}^{i} C_{*}(X) \stackrel{\bar{\tau}}{\longrightarrow} \bar{\tau}^{i+1} C_{*}(X) \longrightarrow 0
$$

where $\iota$ denotes the inclusion.

(3) [10, (3.4) p.124] There is an isomorphism of complexes:

$$
\bar{\sigma} C_{*}(X) \cong C_{*}\left(X / G, X^{G}\right),
$$

where $X^{G}$ is identified with its image in $X / G$.

Proposition 7.2. [10, p.124 (3.7)] If $p>2$ then for any $k \in \mathbb{N}$ there is a commutative diagram of $\mathbb{F}_{p}$-vector spaces with exact rows:

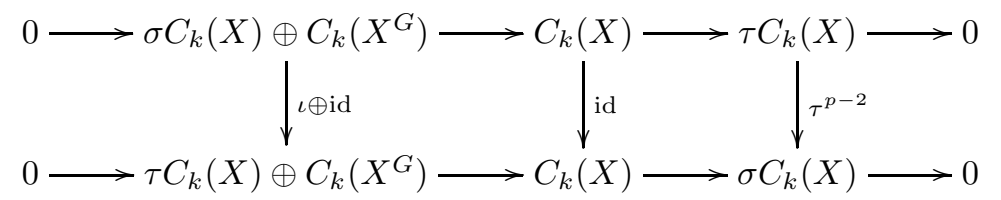


The Smith homology groups are defined by $H_{k}^{\tau^{i}}(X):=H_{k}\left(\bar{\tau}^{i} C_{*}(X)\right)$, the corresponding cohomology groups are $\left.H_{\tau^{i}}^{k}(X)\right)$, whose dimensions over $\mathbb{F}_{p}$ are denoted by $h_{\tau^{i}}^{k}(X)$. We first give some direct consequences of these sequences.

\section{Lemma 7.3.}

(1) If $X^{G} \neq \emptyset$ then $h_{\sigma}^{0}(X)=0$ and $h_{\tau}^{0}(X)=0$.

(2) If $X^{G}=\emptyset$ then $h_{\sigma}^{0}(X)=1$ and $h_{\tau}^{0}(X)=1$.

Proof. First compute for $\sigma$. By Proposition 7.1] (3), we have $H_{\sigma}^{0}(X) \cong H^{0}\left(X / G, X^{G} ; \mathbb{F}_{p}\right)$. From the exact sequence of the pair $\left(X / G, X^{G}\right)$ :

$$
0 \longrightarrow C_{*}\left(X^{G}\right) \longrightarrow C_{*}(X / G) \longrightarrow C_{*}\left(X / G, X^{G}\right) \longrightarrow 0
$$

one gets the exact sequence in cohomology:

$$
0 \longrightarrow H^{0}\left(X / G, X^{G} ; \mathbb{F}_{p}\right) \longrightarrow H^{0}\left(X / G, \mathbb{F}_{p}\right) \stackrel{\iota^{*}}{\rightarrow} H^{0}\left(X^{G}, \mathbb{F}_{p}\right) \longrightarrow \cdots
$$

where $\iota^{*}$ is induced by the inclusion $\iota: X^{G} \hookrightarrow X / G$. Since $X$ is connected, $X / G$ is also connected. If $X^{G} \neq \emptyset$, one has that $\iota^{*} \neq 0$ and $H^{0}\left(X / G, \mathbb{F}_{p}\right) \cong \mathbb{F}_{p}$ so $H_{\sigma}^{0}(X)=0$. Otherwise $H^{0}\left(X / G, X^{G} ; \mathbb{F}_{p}\right) \cong \mathbb{F}_{p}$.

We now compute for $\tau$. Assume that $X^{G} \neq \emptyset$. By Proposition 7.1]2) one gets for all $1 \leq i \leq p-1$ an isomorphism $H_{\tau^{i}}^{0}(X) \cong H_{\tau^{i+1}}^{0}(X)$ so $H_{\tau}^{0}(X)=H_{\sigma}^{0}(X)=0$. If $X^{G}=\emptyset$, by Proposition 7.1(1) one gets the exact sequence:

$$
0 \longrightarrow H_{\tau}^{0}(X) \longrightarrow H^{0}\left(X, \mathbb{F}_{p}\right) \stackrel{\bar{\sigma}^{*}}{\longrightarrow} H_{\sigma}^{0}(X) \longrightarrow \cdots
$$

We show that $\bar{\sigma}^{*}=0$. By Proposition 7.1(1) one has an exact sequence:

$$
0 \longrightarrow H_{\sigma}^{0}(X) \stackrel{\iota^{*}}{\longrightarrow} H^{0}\left(X, \mathbb{F}_{p}\right) \stackrel{\bar{\tau}^{*}}{\longrightarrow} H_{\tau}^{0}(X) \longrightarrow \cdots
$$

so $\iota^{*}$ is injective. The composition $H^{0}\left(X, \mathbb{F}_{p}\right) \stackrel{\bar{\sigma}^{*}}{\rightarrow} H_{\sigma}^{0}(X) \stackrel{\iota^{*}}{\rightarrow} H^{0}\left(X, \mathbb{F}_{p}\right)$ is the action of $\bar{\sigma} \in \mathbb{F}_{p}[G]$. Observe that the action of $g \in G$ on $H^{0}\left(X, \mathbb{F}_{p}\right) \cong \mathbb{F}_{p}$ is trivial since $\mathbb{F}_{p}$ has no order $p$ automorphism, so $\bar{\sigma}$ acts trivially on $H^{0}\left(X, \mathbb{F}_{p}\right)$, that is $\bar{\sigma}^{*} \circ \iota^{*}=0$. Since $\iota^{*}$ is injective, this implies that $\bar{\sigma}^{*}=0$. It follows that $H_{\tau}^{0}(X) \cong H^{0}\left(X, \mathbb{F}_{p}\right) \cong \mathbb{F}_{p}$.

Lemma 7.4. Assume that $X$ is even-dimensional, $H^{\text {odd }}\left(X, \mathbb{F}_{p}\right)=0$ and $X^{G} \neq \emptyset$. Set $2 d:=\operatorname{dim}_{\mathbb{R}} X$. Then:

(1) $h_{\tau}^{1}(X)=h_{\sigma}^{1}(X)=h^{0}\left(X^{G} \mathbb{F}_{p}\right)-1$.

(2) For $0 \leq k \leq d-1$, one has $h_{\tau}^{2 k+1}(X)=h_{\sigma}^{2 k+1}(X)$.

(3) If $\operatorname{dim}_{\mathbb{R}} X^{G} \leq \operatorname{dim}_{\mathbb{R}} X-2$ then:

$$
h_{\sigma}^{2 d-1}(X)=h_{\tau}^{2 d-1}(X)=h_{\sigma}^{2 d}(X)=h_{\tau}^{2 d}(X)=1 .
$$

Proof.

(1) Proposition 7.1 (1) for $i=p-1$ and Lemma 7.3 give the exact sequence:

$$
0 \longrightarrow H^{0}\left(X, \mathbb{F}_{p}\right) \longrightarrow H_{\tau}^{0}(X) \oplus H^{0}\left(X^{G}, \mathbb{F}_{p}\right) \longrightarrow H_{\sigma}^{1}(X) \longrightarrow 0
$$

that implies $h_{\sigma}^{1}(X)=h^{0}\left(X^{G}\right)-1$. By interchanging the roles of $\tau$ and $\sigma$ one gets the second equality.

(2) Similarly, one gets for $0 \leq k \leq d-1$ an exact sequence:

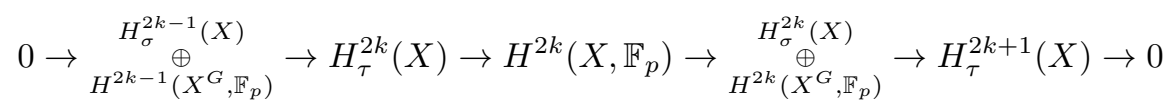


that implies the equality:

$h_{\sigma}^{2 k-1}(X)+h^{2 k-1}\left(X^{G}, \mathbb{F}_{p}\right)-h_{\tau}^{2 k}(X)+h^{2 k}\left(X, \mathbb{F}_{p}\right)-h_{\sigma}^{2 k}(X)-h^{2 k}\left(X^{G}, \mathbb{F}_{p}\right)+h_{\tau}^{2 k+1}(X)=0$.

By interchanging the roles of $\tau$ and $\sigma$ one gets a second equality on dimensions.

Substracting these equalities, one finally obtains:

$$
h_{\sigma}^{2 k-1}(X)-h_{\tau}^{2 k-1}(X)=h_{\sigma}^{2 k+1}(X)-h_{\tau}^{2 k+1}(X) .
$$

Using (1) one concludes.

(3) Clearly $h_{\tau}^{k}(X)=0=h_{\sigma}^{k}(X)$ for $k>2 d$. Proposition 7.1(1) for $i=p-1$ gives the exact sequence:

$$
0 \longrightarrow H_{\sigma}^{2 d-1}(X) \longrightarrow H_{\tau}^{2 d}(X) \longrightarrow H^{2 d}\left(X, \mathbb{F}_{p}\right) \longrightarrow H_{\sigma}^{2 d}(X) \longrightarrow 0
$$

yielding the equality $h_{\sigma}^{2 d-1}(X)-h_{\tau}^{2 d}(X)+1-h_{\sigma}^{2 d}(X)=0$. Proposition 7.1(3) and the exact sequence of the pair $\left(X / G, X^{G}\right)$ gives $h_{\sigma}^{2 d}(X)=1$ so $h_{\sigma}^{2 d-1}(X)=h_{\tau}^{2 d}(X)$. By interchanging the roles of $\sigma$ and $\tau$ one gets an exact sequence:

$$
0 \longrightarrow H_{\tau}^{2 d-1}(X) \longrightarrow H_{\sigma}^{2 d}(X) \longrightarrow H^{2 d}\left(X, \mathbb{F}_{p}\right) \longrightarrow H_{\tau}^{2 d}(X) \longrightarrow 0
$$

that implies that $h_{\tau}^{2 d}(X) \leq 1$. Proposition 7.1(2) for $i=1$ gives an exact sequence:

$$
H_{\tau}^{2 d-1}(X) \longrightarrow H_{\sigma}^{2 d-1}(X) \longrightarrow H_{\tau^{2}}^{2 d}(X) \longrightarrow H_{\tau}^{2 d}(X) \longrightarrow H_{\sigma}^{2 d}(X) \longrightarrow 0
$$

that implies that $h_{\tau}^{2 d}(X) \geq 1$. Finally $h_{\tau}^{2 d}(X)=1$ and one conludes with (2).

Using similar arguments, one can show the following result in the case where the fixed locus is empty:

Lemma 7.5. Assume that $X$ is even-dimensional $\left(2 d:=\operatorname{dim}_{\mathbb{R}} X\right), H^{\text {odd }}\left(X, \mathbb{F}_{p}\right)=0$ and $X^{G}=\emptyset$. Then:

(1) $h_{\tau}^{1}(X)=h_{\sigma}^{1}(X)=1$.

(2) For $0 \leq k \leq d-1$, one has $h_{\tau}^{2 k+1}(X)=h_{\sigma}^{2 k+1}(X)=h^{2 k+1}\left(X / G, \mathbb{F}_{p}\right)$.

(3) $h_{\sigma}^{2 d-1}(X)=h_{\tau}^{2 d-1}(X)=h_{\sigma}^{2 d}(X)=h_{\tau}^{2 d}(X)=1$.

\subsection{A refinement of the Borel-Swan inequality.}

Proposition 7.6. Assume that $p=2, X$ is even-dimensional $\left(2 d:=\operatorname{dim}_{\mathbb{R}} X\right)$, $H^{\text {even }}(X, \mathbb{Z})$ is torsion-free, $H^{\text {odd }}(X, \mathbb{Z})=0, X^{G} \neq \emptyset$ and $\operatorname{dim}_{\mathbb{R}} X^{G} \leq 2 d-2$. Then:

$$
h^{*}\left(X^{G}, \mathbb{F}_{2}\right) \leq h^{*}(X)-2 \sum_{k=1}^{d-1} \mathrm{a}_{G}^{2 k}(X)
$$

with equality if $H^{\text {odd }}\left(X / G, \mathbb{F}_{2}\right)=0$.

Proof. For $1 \leq k \leq d-1$, Proposition 7.1(10) gives exact an sequence:

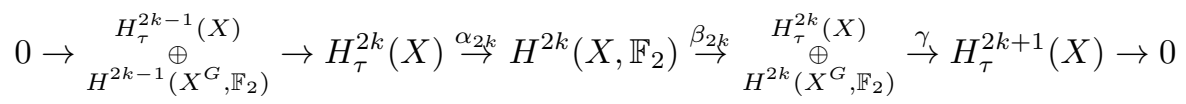

Using $\operatorname{Im}\left(\alpha_{2 k}\right)=\operatorname{Ker}\left(\beta_{2 k}\right)$, this exact sequence cuts into two smaller exact sequences and taking the dimensions one gets the equations:

$$
\left\{\begin{aligned}
h_{\tau}^{2 k-1}(X)+h^{2 k-1}\left(X^{G}, \mathbb{F}_{2}\right)-h_{\tau}^{2 k}(X)+\operatorname{dim} \operatorname{Im}\left(\alpha_{2 k}\right) & =0 \\
\operatorname{dim} \operatorname{Im}\left(\alpha_{2 k}\right)-h^{2 k}(X)+h_{\tau}^{2 k}(X)+h^{2 k}\left(X^{G}, \mathbb{F}_{2}\right)-h_{\tau}^{2 k+1}(X) & =0
\end{aligned}\right.
$$


Summing up these equations, adding the contributions for $1 \leq k \leq d-1$ and using Lemma 7.4(1) one gets:

$$
h^{*}\left(X^{G}, \mathbb{F}_{2}\right)=h^{*}(X)-2 \sum_{k=1}^{d-1} \operatorname{dim} \operatorname{Im}\left(\alpha_{2 k}\right) .
$$

Denote the components by $\beta_{2 k}=\beta_{2 k}^{\prime} \oplus \beta_{2 k}^{\prime \prime}$ and $\gamma=\left(\gamma^{\prime}, \gamma^{\prime \prime}\right)$. Observe that:

$$
\alpha_{2 k} \circ \beta_{2 k}^{\prime}: H^{2 k}\left(X, \mathbb{F}_{2}\right) \rightarrow H^{2 k}\left(X, \mathbb{F}_{2}\right)
$$

is the multiplication by $\bar{\tau}$. For short, we put $\bar{\tau}_{2 k}:=\bar{\tau}_{\mid H^{2 k}\left(X, \mathbb{F}_{2}\right)}$ and we have $\operatorname{Im}\left(\bar{\tau}_{2 k}\right) \subset \operatorname{Im}\left(\alpha_{2 k}\right)$. By Corollary [5.7 $\operatorname{dim} \operatorname{Im}\left(\bar{\tau}_{2 k}\right)=\mathrm{a}_{G}^{2 k}(X)$ so we get the expected inequality.

Take $x \in \operatorname{Im}\left(\alpha_{2 k}\right)$ and write $x=\alpha_{2 k}(y)$ with $y \in H_{\tau}^{2 k}(X)$. Observe that $H^{*}\left(X / G, X^{G} ; \mathbb{F}_{2}\right) \cong H_{\tau}^{*}(X)$ by Proposition [7.1(3) since $\bar{\tau}=\bar{\sigma}$, so that $\gamma^{\prime \prime}$ also appears as the coboundary morphism of the exact sequence of the pair $\left(X / G, X^{G}\right)$ :

$$
\cdots \rightarrow H^{2 k}\left(X^{G}, \mathbb{F}_{2}\right) \stackrel{\gamma^{\prime \prime}}{\rightarrow} H_{\tau}^{2 k+1}(X) \stackrel{\eta}{\rightarrow} H^{2 k+1}\left(X / G, \mathbb{F}_{2}\right) \rightarrow \cdots
$$

Assume that $H^{2 k+1}\left(X / G, \mathbb{F}_{2}\right)=0$. Then $\gamma^{\prime \prime}$ is surjective, so there exists an element $y^{\prime} \in H^{2 k}\left(X^{G}, \mathbb{F}_{2}\right)$ such that $\gamma^{\prime \prime}\left(y^{\prime}\right)=\gamma(y)$. This gives $\gamma\left(y-y^{\prime}\right)=0$ so there exists $z \in H^{2 k}\left(X, \mathbb{F}_{2}\right)$ such that $\beta_{2 k}(z)=y-y^{\prime}$. In particular $\beta_{2 k}^{\prime}(z)=y$. Then $x=\alpha_{2 k} \beta_{2 k}^{\prime}(z)=\bar{\tau}_{2 k}(z)$ giving the equality $\operatorname{Im}\left(\bar{\tau}_{2 k}\right)=\operatorname{Im}\left(\alpha_{2 k}\right)$ and we conclude as before.

Remark 7.7. If $H^{\text {odd }}\left(X / G, \mathbb{F}_{2}\right) \neq 0$, the defect in this inequality can be completely understood by the second inequality:

$$
h^{*}(X)-2 \sum_{k=1}^{d-1} \mathrm{a}_{G}^{2 k}(X)-2 \sum_{k=1}^{d-1} h^{2 k+1}\left(X / G, \mathbb{F}_{2}\right) \leq h^{*}\left(X^{G}, \mathbb{F}_{2}\right) .
$$

To prove this inequality, we keep the notation of the proof, assuming now that $H^{2 k+1}\left(X / G, \mathbb{F}_{2}\right) \neq 0$. Consider the map:

$$
\varphi: H_{\tau}^{2 k}(X) \longrightarrow H^{2 k+1}\left(X / G, \mathbb{F}_{2}\right), \quad y \mapsto \eta \gamma(y) .
$$

If $\varphi(y)=0$, there exists $y^{\prime} \in H^{2 k}\left(X^{G}, \mathbb{F}_{2}\right)$ such that $\gamma(y)=\gamma^{\prime \prime}\left(y^{\prime}\right)$ so $\gamma\left(y-y^{\prime}\right)=0$ and as above there exists $z \in H^{2 k}\left(X, \mathbb{F}_{2}\right)$ such that $\beta_{2 k}^{\prime}(z)=y$. This shows that $\alpha_{2 k}(y) \in \operatorname{Im}\left(\bar{\tau}_{2 k}\right)$. Conversely, if $y \in H_{\tau}^{2 k}(X)$ is such that $\alpha_{2 k}(y) \in \operatorname{Im}\left(\bar{\tau}_{2 k}\right)$, then write $\alpha_{2 k}(y)=\alpha_{2 k} \beta_{2 k}^{\prime}(z)$ with $z \in H^{2 k}\left(X, \mathbb{F}_{2}\right)$. Setting $y^{\prime}:=\beta_{2 k}^{\prime}(z)$, one has $y-y^{\prime} \in \operatorname{Ker}\left(\alpha_{2 k}\right)$. Writting $\beta_{2 k}(z)=\beta_{2 k}^{\prime}(z)+\beta_{2 k}^{\prime \prime}(z)$ and applying $\gamma$ one gets:

$$
\gamma \beta_{2 k}^{\prime}(z)=\gamma^{\prime} \beta_{2 k}^{\prime}(z)=-\gamma^{\prime \prime} \beta_{2 k}^{\prime \prime}(z)
$$

so $\eta \gamma\left(y^{\prime}\right)=0$. This shows that $\alpha_{2 k}^{-1}\left(\operatorname{Im}\left(\bar{\tau}_{2 k}\right)\right)=\operatorname{Ker}(\varphi)+\operatorname{Ker}\left(\alpha_{2 k}\right)$. In particular, the map $\alpha_{2 k}$ induces a surjection:

$$
H_{\tau}^{2 k}(X) / \operatorname{Ker}(\varphi) \rightarrow \operatorname{Im}\left(\alpha_{2 k}\right) / \operatorname{Im}\left(\bar{\tau}_{2 k}\right) .
$$

Computing the dimensions, one gets:

$$
\operatorname{dim} \operatorname{Im}\left(\alpha_{2 k}\right)-\operatorname{dim} \operatorname{Im}\left(\bar{\tau}_{2 k}\right) \leq \operatorname{dim}\left(H_{\tau}^{2 k}(X) / \operatorname{Ker}(\varphi)\right) \leq h^{2 k+1}(X / G) .
$$

We thus proved the inequalities:

$$
\operatorname{dim} \operatorname{Im}\left(\bar{\tau}_{2 k}\right) \leq \operatorname{dim} \operatorname{Im}\left(\alpha_{2 k}\right) \leq \operatorname{dim} \operatorname{Im}\left(\bar{\tau}_{2 k}\right)+h^{2 k+1}(X / G) .
$$

and we conclude as above. 
Proposition 7.8. Assume that $3 \leq p \leq 19, X$ is even-dimensional $\left(2 d:=\operatorname{dim}_{\mathbb{R}} X\right)$, $H^{\text {even }}(X, \mathbb{Z})$ is torsion-free, $H^{\text {odd }}(X, \mathbb{Z})=0, X^{G} \neq \emptyset$ and $\operatorname{dim}_{\mathbb{R}} X^{G} \leq 2 d-2$. Then:

$$
h^{*}\left(X^{G}, \mathbb{F}_{p}\right) \leq h^{*}(X)-2 \sum_{k=1}^{d-1} \mathrm{a}_{G}^{2 k}(X)-(p-2) \sum_{k=1}^{d-1} \mathrm{~m}_{G}^{2 k}(X)
$$

with equality if $H^{\text {odd }}\left(X / G, \mathbb{F}_{p}\right)=0$.

Proof. For $1 \leq k \leq d-1$, Proposition 7.1(11) with $i=1$ gives exact an sequence:

$$
0 \rightarrow \underset{H^{2 k-1}\left(X^{G}, \mathbb{F}_{p}\right)}{H_{\tau}^{2 k-1}(X)} \rightarrow H_{\sigma}^{2 k}(X) \stackrel{\alpha_{2 k}}{\rightarrow} H^{2 k}\left(X, \mathbb{F}_{p}\right) \stackrel{\beta_{2 k}}{\rightarrow} \underset{H^{2 k}\left(X^{G}, \mathbb{F}_{p}\right)}{\stackrel{H_{\tau}^{2 k}(X)}{\rightarrow}} \stackrel{\stackrel{\gamma}{\rightarrow}}{H_{\sigma}^{2 k+1}}(X) \rightarrow 0
$$

Using $\operatorname{Im}\left(\alpha_{2 k}\right)=\operatorname{Ker}\left(\beta_{2 k}\right)$ one gets the equations:

$$
\left\{\begin{array}{r}
\operatorname{dim} \operatorname{Im}\left(\alpha_{2 k}\right)-h_{\sigma}^{2 k}(X)+h_{\tau}^{2 k-1}(X)+h^{2 k-1}\left(X^{G}, \mathbb{F}_{p}\right)=0 \\
\operatorname{dim} \operatorname{Im}\left(\alpha_{2 k}\right)-h^{2 k}(X)+h_{\tau}^{2 k}(X)+h^{2 k}\left(X^{G}, \mathbb{F}_{p}\right)-h_{\sigma}^{2 k+1}(X)=0
\end{array}\right.
$$

Summing up these equations, adding the contributions for $1 \leq k \leq d-1$ and using Lemma 7.4 one gets:

$$
h^{*}\left(X^{G}, \mathbb{F}_{2}\right)=h^{*}(X)+\sum_{k=1}^{d-1}\left(h_{\sigma}^{2 k}(X)-h_{\tau}^{2 k}(X)\right)-2 \sum_{k=1}^{d-1} \operatorname{dim} \operatorname{Im}\left(\alpha_{2 k}\right) .
$$

Exchanging the roles of $\tau$ and $\sigma$ (Proposition 7.1(1) with $i=p-1$ ) one gets a similar exact sequence, where we denote by $\widetilde{\alpha}, \widetilde{\beta}$ the corresponding maps. The same computation gives:

$$
\operatorname{dim} \operatorname{Im}\left(\widetilde{\alpha}_{2 k}\right)-h_{\tau}^{2 k}(X)+h_{\sigma}^{2 k-1}(X)+h^{2 k-1}\left(X^{G}, \mathbb{F}_{p}\right)=0
$$

so we get the relation:

$$
\operatorname{dim} \operatorname{Im}\left(\alpha_{2 k}\right)-\operatorname{dim} \operatorname{Im}\left(\widetilde{\alpha}_{2 k}\right)=h_{\sigma}^{2 k}(X)-h_{\tau}^{2 k}(X) .
$$

This gives:

$$
h^{*}\left(X^{G}, \mathbb{F}_{2}\right)=h^{*}(X)-\sum_{k=1}^{d-1} \operatorname{dim} \operatorname{Im}\left(\alpha_{2 k}\right)-\sum_{k=1}^{d-1} \operatorname{dim} \operatorname{Im}\left(\widetilde{\alpha}_{2 k}\right) .
$$

Denote the components by $\beta_{2 k}=\beta_{2 k}^{\prime} \oplus \beta_{2 k}^{\prime \prime}$ and $\widetilde{\beta}_{2 k}=\widetilde{\beta}_{2 k}^{\prime} \oplus \widetilde{\beta}_{2 k}^{\prime \prime}$. Note that $\alpha_{2 k} \circ \widetilde{\beta}_{2 k}^{\prime}$ is the multiplication by $\bar{\sigma}$ in $H^{2 k}\left(X, \mathbb{F}_{p}\right)$, denoted $\bar{\sigma}_{2 k}$, whereas $\widetilde{\alpha}_{2 k} \circ \beta_{2 k}^{\prime}$ is the multiplication by $\bar{\tau}$ in $H^{2 k}\left(X, \mathbb{F}_{p}\right)$, denoted $\bar{\tau}_{2 k}$. This shows that $\operatorname{Im}\left(\bar{\sigma}_{2 k}\right) \subset \operatorname{Im}\left(\alpha_{2 k}\right)$ and $\operatorname{Im}\left(\bar{\tau}_{2 k}\right) \subset \operatorname{Im}\left(\widetilde{\alpha}_{2 k}\right)$. By Corollary 5.7 one has $\operatorname{dim} \operatorname{Im}\left(\bar{\sigma}_{2 k}\right)=\mathrm{a}_{G}^{2 k}(X)$, and by Proposition 5.1 and Corollaries 5.7 \& 5.10 one has:

$$
\begin{aligned}
\operatorname{dim} \operatorname{Im}\left(\bar{\tau}_{2 k}\right) & =h^{2 k}(X)-\operatorname{dim} H^{2 k}\left(X, \mathbb{F}_{p}\right)^{G} \\
& =(p-1) \ell_{p}^{2 k}(X)+(p-2) \ell_{p-1}^{2 k}(X) \\
& =\mathrm{a}_{G}^{2 k}(X)+(p-2) \mathrm{m}_{G}^{2 k}(X),
\end{aligned}
$$

hence the expected inequality. 
Assume that $H^{\text {odd }}\left(X / G, \mathbb{F}_{p}\right)=0$. For $1 \leq k \leq d-1$, Proposition 7.2 gives a commutative diagram with exact rows:

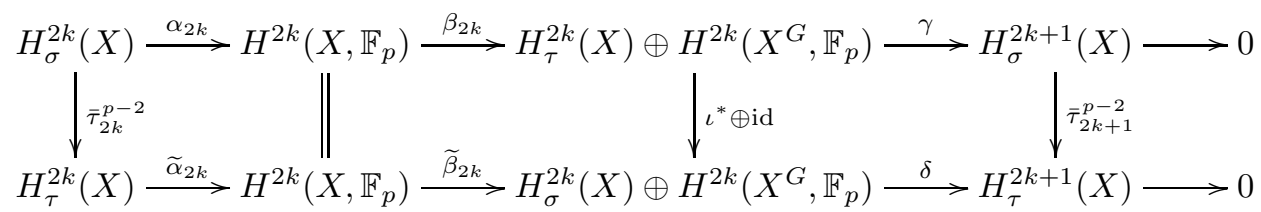

We first show that $\bar{\tau}_{2 k+1}^{p-2}$ is injective by a diagram chasing. Denote by $\gamma=\left(\gamma^{\prime}, \gamma^{\prime \prime}\right)$ the components. As previously observed, since $H^{2 k+1}\left(X / G, \mathbb{F}_{p}\right)=0 \gamma^{\prime \prime}$ is surjective. Let $x \in H_{\sigma}^{2 k+1}(X)$ such that $\bar{\tau}_{2 k+1}^{p-2}(x)=0$. There exists $y \in H^{2 k}\left(X^{G}, \mathbb{F}_{p}\right)$ such that $\gamma(y)=\gamma^{\prime \prime}(y)=x$. Considering $y \in H^{2 k}\left(X^{G}, \mathbb{F}_{p}\right)$ in the second row of the diagram, one gets $\delta(y)=0$ hence there exists $z \in H^{2 k}\left(X, \mathbb{F}_{p}\right)$ such that $\widetilde{\beta}_{2 k}(z)=\widetilde{\beta}_{2 k}^{\prime \prime}(z)=y$. Considering $z$ in the first row, one gets $\beta_{2 k}(z)=\beta_{2 k}^{\prime \prime}(z)=y$. Hence $x=\gamma(y)=\gamma \beta_{2 k}(z)=0$. By Lemma 7.4(2), we deduce that $\bar{\tau}_{2 k+1}^{p-2}$ is an isomorphism.

We deduce that the map denoted $\iota^{*}$ in the diagram is surjective. Take $x \in H_{\sigma}^{2 k}(X)$. The element $\delta(x)$ admits a preimage $y$ by $\bar{\tau}_{2 k+1}^{p-2}$. Since $\gamma^{\prime \prime}$ is surjective, there exists $z \in H^{2 k}\left(X^{G}, \mathbb{F}_{p}\right)$ such that $\gamma^{\prime \prime}(z)=y$. Considering $z$ in the second row, one gets $\delta(z)=\bar{\tau}_{2 k+1}^{p-2}(y)=\delta(x)$, hence $x-z \in \operatorname{Ker}(\delta)=\operatorname{Im}\left(\widetilde{\beta}_{2 k}\right)$ so there exists $y$ such that $\widetilde{\beta}_{2 k}^{\prime}(y)=x$ and $\widetilde{\beta}_{2 k}^{\prime \prime}(y)=-z$. Considering $y$ in the first row, one gets $\iota^{*} \beta_{2 k}^{\prime}(y)=\widetilde{\beta}_{2 k}^{\prime}(y)=x$.

We deduce that $\operatorname{Im}\left(\alpha_{2 k}\right) \subset \operatorname{Im}\left(\bar{\sigma}_{2 k}\right)$. Take $y=\alpha_{2 k}(x)$. Considering $x \in H_{\sigma}^{2 k}(X)$ in the second row, since $\iota^{*}$ is surjective there exists $w \in H_{\tau}^{2 k}(X)$ such that $\iota^{*}(w)=x$. Since $\gamma^{\prime \prime}$ is surjective, there exists $t \in H^{2 k}\left(X^{G}, \mathbb{F}_{p}\right)$ with $\gamma^{\prime \prime}(t)=\gamma(w)$, hence $\gamma(w-t)=0$ so there exists $z \in H^{2 k}\left(X, \mathbb{F}_{p}\right)$ such that $\beta_{2 k}^{\prime}(z)=w$ and $\beta_{2 k}^{\prime \prime}(z)=-t$. Considering $z$ in the second row, one gets $\widetilde{\beta}_{2 k}^{\prime}(z)=\iota^{*} \beta_{2 k}^{\prime}(z)=\iota^{*}(w)=x$ so $y=\alpha_{2 k} \widetilde{\beta}_{2 k}^{\prime}(z)=\bar{\sigma}_{2 k}(z)$.

To conclude, we show that $\operatorname{Im}\left(\widetilde{\alpha}_{2 k}\right) \subset \operatorname{Im}\left(\bar{\tau}_{2 k}\right)$. Take $y=\widetilde{\alpha}_{2 k}(x)$. Considering $x$ in the first row, since $\gamma^{\prime \prime}$ is surjective there exists $w$ such that $\gamma(x)=\gamma^{\prime \prime}(w)=\gamma(w)$, so there exists $z$ with $\beta_{2 k}^{\prime}(z)=x$ and $\beta_{2 k}^{\prime \prime}(z)=-w$, hence $y=\widetilde{\alpha}_{2 k} \beta_{2 k}^{\prime}(z)=\bar{\tau}_{2 k}(z)$.

The expected equality follows.

\section{REFERENCES}

1. C. Allday and V. Puppe, Cohomological methods in transformation groups, Cambridge Studies in Advanced Mathematics, vol. 32, Cambridge University Press, Cambridge, 1993. MR 1236839 (94g:55009)

2. M. Artebani, A. Sarti, and S. Taki, Non-symplectic automorphisms of prime order on K3 surfaces, Math. Z. (to appear) 268 (2011), 507-533.

3. A. Beauville, Some remarks on Kähler manifolds with $c_{1}=0$, Classification of algebraic and analytic manifolds (Katata, 1982), Progr. Math., vol. 39, Birkhäuser Boston, Boston, MA, 1983, pp. 1-26.

4. — Variétés Kähleriennes dont la première classe de Chern est nulle, J. Differential Geom. 18 (1983), no. 4, 755-782 (1984).

5. A. Beauville and R. Donagi, La variété des droites d'une hypersurface cubique de dimension 4, C. R. Acad. Sci. Paris Sér. I Math. 301 (1985), no. 14, 703-706.

6. S. Boissière, Automorphismes naturels de l'espace de Douady de points sur une surface, Canad. J. Math. 64 (2012), 3-23. 
7. S. Boissière, M. Nieper-Wißkirchen, and A. Sarti, Higher dimensional Enriques varieties and automorphisms of generalized kummer varieties, J. Math. Pures Appl. 95 (2011), 553-563.

8. A. Borel, Seminar on transformation groups, With contributions by G. Bredon, E. E. Floyd, D. Montgomery, R. Palais. Annals of Mathematics Studies, No. 46, Princeton University Press, Princeton, N.J., 1960.

9. H. Brandt and O. Intrau, Tabellen reduzierter positiver ternärer quadratischer Formen, Abh. Sächs. Akad. Wiss. Math.-Nat. Kl. 45 (1958), no. 4, 261.

10. G. E. Bredon, Introduction to compact transformation groups, Academic Press, New York, 1972, Pure and Applied Mathematics, Vol. 46

11. K. S. Brown, Cohomology of groups, Graduate Texts in Mathematics, vol. 87, Springer-Verlag, New York, 1994, Corrected reprint of the 1982 original. MR 1324339 (96a:20072)

12. C. W. Curtis and I. Reiner, Representation theory of finite groups and associative algebras, Wiley Classics Library, John Wiley \& Sons Inc., New York, 1988, Reprint of the 1962 original, A Wiley-Interscience Publication. MR 1013113 (90g:16001)

13. P. Deligne, Théorème de Lefschetz et critères de dégénérescence de suites spectrales, Inst. Hautes Études Sci. Publ. Math. (1968), no. 35, 259-278.

14. W. G. Dwyer and C. W. Wilkerson, Smith theory revisited, Ann. of Math. (2) 127 (1988), no. 1, 191-198.

15. A. D. Elagin, On an equivariant derived category of bundles of projective spaces, Tr. Mat. Inst. Steklova 264 (2009), no. Mnogomernaya Algebraicheskaya Geometriya, 63-68.

16. G. Ellingsrud, L. Göttsche, and M. Lehn, On the cobordism class of the Hilbert scheme of a surface, J. Algebraic Geom. 10 (2001), no. 1, 81-100.

17. A. Fujiki, On the de Rham cohomology group of a compact Kähler symplectic manifold, Algebraic geometry, Sendai, 1985, Adv. Stud. Pure Math., vol. 10, North-Holland, Amsterdam, 1987, pp. 105-165.

18. A. Garbagnati and A. Sarti, Symplectic automorphisms of prime order on K3 surfaces, J. Algebra 318 (2007), no. 1, 323-350.

19. B. Hassett, Special cubic fourfolds, Compositio Math. 120 (2000), no. 1, 1-23.

20. D. Huybrechts, Compact hyper-Kähler manifolds: basic results, Invent. Math. 135 (1999), no. $1,63-113$.

21. V. M. Kharlamov, The topological type of nonsingular surfaces in $\mathbf{R} P^{3}$ of degree four, Funct. Anal. Appl. 10 (1976), no. 4, 295-304.

22. V. A. Krasnov, Harnack-Thom inequalities for mappings of real algebraic varieties, Izv. Akad. Nauk SSSR Ser. Mat. 47 (1983), no. 2, 268-297.

23. ㄴ Real algebraic GM-manifolds, Izv. Ross. Akad. Nauk Ser. Mat. 62 (1998), no. 3, 39-66.

24. M. Lehn and C. Sorger, The cup product of Hilbert schemes for K3 surfaces, Invent. Math. 152 (2003), no. 2, 305-329.

25. E. Markman, The Beauville-Bogomolov class as a characteristic class, arXiv:1105:3223v1.

26. _ A survey of Torelli and monodromy results for holomorphic-symplectic varieties, arXiv:1101.4606v2.

27. Integral generators for the cohomology ring of moduli spaces of sheaves over Poisson surfaces, Adv. Math. 208 (2007), no. 2, 622-646.

28. - Integral constraints on the monodromy group of the hyperKähler resolution of a symmetric product of a K3 surface, Internat. J. Math. 21 (2010), no. 2, 169-223.

29. D. Markushevich, Rational Lagrangian fibrations on punctual Hilbert schemes of K3 surfaces, Manuscripta Math. 120 (2006), no. 2, 131-150.

30. J. M. Masley and H. L. Montgomery, Cyclotomic fields with unique factorization, J. Reine Angew. Math. 286/287 (1976), 248-256.

31. G. Mongardi, On symplectic automorphisms of hyperkähler fourfolds, arXiv:1112.5073v3.

32. - Symplectic involutions on deformations of $K 3^{[2]}$, arXiv:1107.2854.

33. H. Nakajima, Heisenberg algebra and Hilbert schemes of points on projective surfaces, Ann. of Math. (2) 145 (1997), no. 2, 379-388.

34. M. Nieper-Wißkirchen, Chern numbers and Rozansky-Witten invariants of compact hyperKähler manifolds, World Scientific Publishing Co. Inc., River Edge, NJ, 2004.

35. V. V. Nikulin, Finite groups of automorphisms of Kählerian K3 surfaces, Trudy Moskov. Mat. Obshch. 38 (1979), 75-137. 
36. - Integral symmetric bilinear forms and some of their geometric applications, Izv. Akad. Nauk SSSR Ser. Mat. 43 (1979), no. 1, 111-177.

37. Kieran G. O'Grady, Irreducible symplectic 4-folds numerically equivalent to $(K 3)^{[2]}$, Commun. Contemp. Math. 10 (2008), no. 4, 553-608.

38. K. Oguiso and S. Schröer, Enriques manifolds, J. Reine Angew. Math. (to appear) (2011).

39. Z. Qin and W. Wang, Integral operators and integral cohomology classes of Hilbert schemes, Math. Ann. 331 (2005), no. 3, 669-692.

40. M. Verbitsky, Cohomology of compact hyper-Kähler manifolds and its applications, Geom. Funct. Anal. 6 (1996), no. 4, 601-611.

Samuel Boissière, Laboratoire de Mathématiques et Applications, UmR CNRS 6086, Université de Poitiers, Téléport 2, Boulevard Marie et Pierre Curie, F-86962 FuturoSCOpe Chasseneuil

E-mail address: samuel.boissiere@math.univ-poitiers.fr

URL: http://www-math.sp2mi.univ-poitiers.fr/ sboissie/

Marc Nieper-Wisskirchen, Lehrstuhl für Algebra und Zahlentheorie, UniversitätsStrasse 14, D-86159 Augsburg

E-mail address: marc.nieper-wisskirchen@math.uni-augsburg.de

$U R L:$ http://www.math.uni-augsburg.de/alg/

Alessandra Sarti, Laboratoire de Mathématiques et Applications, UMR CNRS 6086, Université de Poitiers, Téléport 2, Boulevard Marie et Pierre Curie, F-86962 FuturoSCOPE Chasseneuil

E-mail address: alessandra.sarti@math.univ-poitiers.fr

URL: http://www-math.sp2mi.univ-poitiers.fr/ sarti/ 